\title{
Über den Einfluß von Rotschlamm auf die Kultur einiger mariner Planktonalgen
}

\author{
H. KAYSER \\ Biologische Anstalt Helgoland (Meeresstation); \\ Helgoland, Bundesrepublik Deutscbland
}

\begin{abstract}
On the effect of red mud on the culture of some marine plankton algae. "Red mud" originates from bauxite processing, the first step of aluminium production. A newly constructed plant intends to release about 800000 tons of this waste product into the southern North Sea. The effect of red mud on the culture of some marine algae was investigated. The dinoflagellates Peridinium trochoideum, Prorocentrum micans and Gymnodinium splendens, and the diatoms Coscinodiscus granii and Chaetoceros socialis served as test organisms. They were examined in batch cultures and in continuous cultures (turbidostats) to which 0.001 to $50 \mathrm{~g}$ of red mud per 1 sea water was added. Multiplication rates of the algae were chosen as criterion for assessing the influence of red mud. Maximum cell densities of the batch cultures were also determined. When red mud was added to the batch cultures once, at the beginning of the experiments, the test algae exhibited initial reductions in population growth at 0.005 up to $0.5 \mathrm{~g}$ of red mud/1 sea water, depending on the species. In most experiments they recovered from initial growth-rate reduction and grew to nearly the same cell densities as did the controls. In the continuous cultures, suspended red mud was added daily. After 9 days, this caused irreversible break-down of Peridinium trocboideum populations at $0.01 \mathrm{~g}$ red mud/1/day. Coscinodiscus granii populations were irreversibly damaged at $0.05 \mathrm{~g} / 1 / \mathrm{day}$; Prorocentrum micans, at $0.5 \mathrm{~g} / 1 /$ day. All test algae represent euryplastic forms. Other, more stenoplastic planktonic algae species are likely to be less tolerant to red mud exposure.
\end{abstract}

\section{EINLEITUNG}

Züchtungsexperimente mit marinen planktischen Algen bieten die Möglichkeit, die

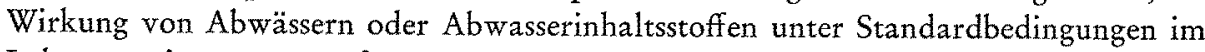
Laborexperiment zu prüfen. Seit einigen Jahren werden daher an der Biologischen Anstalt Helgoland entsprechende Testmethoden entwickelt und durchgeführt. Die Bestimmung der Vermehrungsrate geeigneter Testalgen dient dabei zum Nachweis erster, noch subletaler Abwassereinfliisse. Als weitere Kriterien werden die maximal erreichbaren Zelldichten der Testkulturen und die Mortalität der Algen unter Abwassereinfluß herangezogen. Erste Ergebnisse für die Anwendung des Testverfahrens wurden bei der Untersuchung der Abwässer eines Titandioxidwerkes gewonnen (KAYSER 1969, $1970 \mathrm{a}, 1970 \mathrm{~b}, 1971 \mathrm{a}, \mathrm{b})$. Dieses überwiegend aus $10 \%$ Schwefelsäure und $14 \%$ Eisensulfat bestehende Abwasser wird seit Mai 1969 in Mengen von täglich etwa 1800 t in ein Versenkungsgebiet $14 \mathrm{sm}$ nordwestlich von Helgoland mit Hilfe von Spezialtankern in die Nordsee eingebracht (WEICHART 1969). 
In der vorliegenden Arbeit wird über Versuche zur Ermittlung des Einflusses von Rotschlamm auf marine Planktonalgen berichtet. Rotschlamm ist ein Abfallprodukt, das bei der Aluminiumoxidgewinnung aus Bauxit in großen Mengen anfällt. Der Antrag der Vereinigte Aluminiumwerke AG Berlin-Bonn, ab 1973 Rotschlamm in Mengen von zunächst ca. $800000 \mathrm{t}$ (Naßgewicht) jährlich in der südlichen Nordsee zu versenken, gab den unmittelbaren Anlaß zur Aufnahme großangelegter Versuche über die $\mathrm{zu}$ erwartenden Auswirkungen. An diesen Untersuchungen beteiligten sich die Biologische Anstalt Helgoland, die Bundesforschungsanstalt für Fischerei, das Deutsche Hydrographische Institut und das Institut für Meeresforschung, Bremerhaven. Neben Laborexperimenten an Meeresorganismen wurde auch ein Großversuch in See durchgeführt, bei dem Wirkung und Verbleib von $15000 \mathrm{t}$ Rotschlamm untersucht wurden. Einzelergebnisse der Versuche wurden von Apelt (1971), Dethlefsen (1972a, b), Greve \& Kinne (1971), Halsband \& Halsband (1971), Halsband (1971), Kayser (1971b und im Druck), Nauke (im Druck), Paffenhöfer (1971, 1972), Rachor (1971), Rosenthal (1971) und Rosenthal et al. (1973) und ein kritischer Obersichtsartikel von DETHLEFSEN \& ROSENTHAL (1973) veröffentlicht.

\section{MATERIAL UND METHODE}

Der für die Versuche verwendete Rotschlamm wurde von der Vereinigte Aluminium Werke AG (VAW) zur Verfügung gestellt. Er hat schlammig- bis stichfeste Konsistenz, ist intensiv rotbraun gefärbt und reagiert alkalisch ( $\mathrm{pH} \mathrm{12,2).} \mathrm{Nach} \mathrm{Angaben}$ des Werkes hat der produzierte Rotschlamm folgende Zusammensetzung:

\begin{tabular}{|c|c|c|c|}
\hline $\mathrm{Al}_{2} \mathrm{O}_{3}$ & $=31,9 \%$ & $\mathrm{Na}{ }_{2} \mathrm{O}$ (gebunden) & $=10,3 \%$ \\
\hline $\mathrm{Al}_{2} \mathrm{O}_{3}$ (wasserlösl.) & $=0,5 \%$ & $\mathrm{Na}_{2} \mathrm{O}$ (wasserlösl.) & $0,9 \%$ \\
\hline $\mathrm{Fe}_{2} \mathrm{O}_{3}$ & $=20,7 \%$ & $\mathrm{TiO}_{2}$ & $7,4 \%$ \\
\hline $\mathrm{SiO}_{2}$ & $=16,6 \%$ & 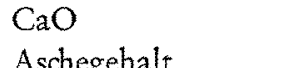 & $\begin{array}{l}=0,7 \% \\
=1100 \%\end{array}$ \\
\hline
\end{tabular}

Je nach Herkunft des verwendeten Bauxits kann die Zusammensetzung leicht variieren. Eine röntgenographische Analyse der VAW ergab folgende mineralogische Zusammensetzung: Eisenmineralien: Haematit, Lepidocrocit; Aluminiummineralien: Böhmit, Hydrargillit; Titanmineralien: Anatas, Rutil; Siliziummineralien: Quarz; Silikate: Hydroxylsodalith.

Die Korngröße liegt in der Regel bei 4-8 $\mu \mathrm{m}$. Etwa 50\% der Partikel sind kleiner als $18 \mu \mathrm{m}$, nur $0,5 \%$ größer als $300 \mu \mathrm{m}$. Das Material fiel bei der Gewinnung von Aluminiumoxid aus Weipa-Bauxit an.

Als Testalgen wurden die Dinoflagellaten Peridinium trochoideum, Prorocentrum micans und Gymnodinium splendens sowie die Diatomeen Coscinodiscus granii und Chaetoceros socialis verwendet. Alle fünf Algenarten kommen zeitweise häufig in der Nordsee vor und wurden aus dem Plankton isoliert*. Die Kulturen wurden unialgal,

* Herrn Dr. Drebes (BAH) danke ich für die Überlassung der Kulturen von Peridinium trochoideum und Gymnodinium splendens. 
jedoch nicht bakterienfrei gehalten. Die Versuche wurden in temperaturkonstanten Räumen durchgeführt. Die Wassertemperatur in den Versuchsgefäßen betrug $18 \pm$ $2^{\circ} \mathrm{C}$. Die Beleuchtung der Kulturen erfolgte durch seitlich angeordnete Tageslichtleuchtröhren (Osram-L $40 \mathrm{~W} / 15$ ) mit einer Lichtintensität von zusammen etwa 6000 Lux. Der Abstand der Lichtquellen von den Gefäßen betrug $6 \mathrm{~cm}$. Ein Tag-NachtRhythmus von 14 Stunden Licht und 10 Stunden Dunkelheit wurde eingehalten. Für die Bestimmung der Zelldichten wurde ein Plankton-Mikroskop nach UTERMöHL verwendet.

Die Züchtung der Algen erfolgte nach zwei Verfahren: (1) Batch-Kulturen und (2) Kontinuierliche Kulturen nach dem Turbidostat-Prinzip. Die Versuchsbedingungen wurden in den einzelnen Versuchsserien jeweils leicht variiert. In der folgenden Aufstellung wird eine Übersicht über die verschiedenen Versuchsanordnungen gegeben.

(1) B a t ch-Kulture n. Die Algen wurden in 1,5-I-, 2-1- und 5-1-Gefäßen gezüchtet. Als Versuchsmedium diente für die beiden Diatomeenarten sterilfiltriertes Seewasser der Helgoländer Reede $(32 \% \pm 1,5 \%$ S, Millipore-Filter von $0,02 \mu \mathrm{m}$ Porenweite). Für die Kultur der Dinoflagellaten wurde diesem Seewasser 0,1 g Na$\mathrm{NO}_{3}$ und $0,02 \mathrm{~g} \mathrm{NaH}_{2} \mathrm{PO}_{4}$ pro Liter Seewasser (Schreiberlösung) zugesetzt. In allen Versuchsgefäßen wurde durch grobblasige Belüftung (ca. $40 \mathrm{ml} \mathrm{Luft/min)} \mathrm{eine}$ schwache Turbulenz des Kulturmediums aufrechterhalten.

(a) Standversuche: Rotschlamm wurde den Kulturen einmalig am ersten Versuchstag in fein suspendierter Form zugesetzt. Trotz der leichten Turbulenz des Kulturmediums sedimentierte er zum größten Teil im Verlauf einiger Stunden bis Tage am Boden der Versuchsgefäße und wurde während der ganzen Versuchsdauer nicht wieder aufgewirbelt.

(b) Schüttelversuche: Der den Kulturen am ersten Versuchstage einmalig in fein suspendierter Form zugesetzte Rotschlamm wurde während der ganzen Versuchsdauer zweimal täglich jeweils morgens und abends von Hand aufgeschüttelt.

(c) Rührwerksversuche: Der den Kulturen einmalig bei Versuchsbeginn fein suspendiert zugesetzte Rotschlamm wurde während der ganzen Versuchszeit kontinuierlich durch Magnetrührwerke soweit wie möglich in Turbulenz gehalten.

(d) Überstandsversuche: Die Algen (Prorocentrum micans) wurden im abdekantierten Uberstandswasser einer Rotschlammprobe gezüchtet. Hierfür wurde $250 \mathrm{~g}$ Rotschlamm fein suspendiert in 11 Seewasser eingebracht und vermischt. Nach eintägiger Sedimentation in ruhender Wassersäule wurde die überstehende Lösung abdekantiert und für den Versuch verwendet. In einer anderen Serie wurden die Algen (Gymnodinium splendens) den Kulturgefäßen erst dann zugesetzt, nachdem der bei Versuchsbeginn fein suspendiert eingegebene Rotschlamm über zwei Tage sedimentiert war. Dieser wurde während der ganzen Versuchsdauer nicht wieder aufgewirbelt.

(2) Kontinuie $\mathrm{r}$ lich e $\mathrm{Kult}$ u r e n. Die Versuche wurden nach dem Turbidostat-Prinzip in 1-1-Gefäßen und in einem 8-1-Fermenter der Firma Eschweiler, Kiel, durchgeführt. Die Dinoflagellaten wurden in Schreiberlösung, die Diatomee Coscinodiscus granii in einer mit $0,015 \mathrm{~g} \mathrm{Na}_{2} \mathrm{SiO}_{3} \cdot 9 \mathrm{H}_{2} \mathrm{O}$ (pro Liter) angereicherten Schreiberlösung gezüchtet. Alle Kulturgefäße wurden grobblasig belüftet (ca. $40 \mathrm{ml}$ Luft/min). In den Kontrollkulturen wurde die Zelldichte der in exponentieller Phase wachsenden Algen durch kontinuierliches Zudosieren von Nährlösung und Absaugen 
einer entsprechenden Menge zellhaltiger Kulturlösung auf einem willkürlich festgesetzten Wert konstant gehalten. Hierfür wurde die Dichte der Kontrollkultur mehrmals am Tag bestimmt und mit Hilfe des Durchflusses (Regulierung der Dosierpumpe) genau auf den vorgesehenen Sollwert eingestellt. Der Durchsatz entsprach dabei jeweils genau der Teilungsrate der Algen und wurde für jede Art in Vorversuchen bestimmt.

Rotschlamm wurde den Testkulturen unabhängig vom Durchsatz des Kulturmediums während der ganzen Versuchszeit täglich einmal in fein suspendierter Form von Hand zugesetzt und nach seiner Sedimentation nicht wieder aufgewirbelt. Da in den Testkulturen der gleiche Durchsatz an Nährlösung eingehalten wurde wie in der Kontrollkultur, machte sich eine Schadwirkung durch Rotschlamm in einem Absinken der Zelldichte im Vergleich zur zellkonstanten Kontrolle bemerkbar. Für die Förderung des Kulturmediums wurden zwei Ismatec-Microschlauchpumpen mit je 13 Kanälen verwendet.

\section{ERGEBNISSE}

Die Ergebnisse der Kulturversuche sind im folgenden in der Weise dargestellt, daß die mit einer Algenart durchgeführten Experimente jeweils in einem Abschnitt zusammengefaßt sind. Diese Darstellung erleichtert den Vergleich der an einer Art mit unterschiedlichen Versuchsmethoden gewonnenen Resultate. Ein Vergleich der Ergebnisse der Algenarten untereinander folgt im Kapitel „Diskussion“.

\section{Peridinium trochoideum}

\section{Batch-Kulturen}

Schüttelversuche. Die Algen wurden in sechs Kulturgefäßen mit je 1,5 1 Inhalt mit einer Anfangszelldichte von je $100 \mathrm{Zellen} / \mathrm{ml}$ angesetzt. $\mathrm{Zu}$ fünf Gefäßen wurde bei Versuchsbeginn Rotschlamm in Mengen von $0,01,0,05,0,1,0,5$ und $1 \mathrm{~g} / 1$ Seewasser zugesetzt und täglich im Verlauf des Versuchs zweimal aufgeschüttelt (vgl. p. 359). Auf Grund der schwachen Beliiftung der Kulturen verteilte sich der Rotschlamm unmittelbar nach der Zugabe zunächst gleichmäßig im ganzen Versuchsgefäß. Schon nach einer halben Stunde zeigte es sich, daß die Rotschlammpartikel großenteils zu größeren, lockeren Aggregationen zusammentraten (50-150 $\mu \mathrm{m}$ Durchmesser) und zu sedimentieren begannen. Diese stark adhäsiven Eigenschaften frisch in Seewasser gebrachten Rotschlammes sind vermutlich auf Ausfällungen gelöster Aluminiumverbindungen aus der alkalischen Restlauge (Porenlösung) des Rotschlammes zurückzuführen, die sich bei der Neutralisation im Seewasser bilden (Rosenthal et al. 1973). Die Sedimentation des Rotschlammes erfolgte relativ langsam. Nach den Versuchen von PAFFenhöfER (1972) ist in bewegtem Seewasser zehn Stunden nach Zugabe noch etwa $21 \%$ des Rotschlammes in Schwebe. Nach 40 Stunden ist die Rotschlammkonzentration in der Wassersäule auf etwa $9 \%$ abgesunken. Als erstes sedimentieren die größeren Rotschlammaggregationen; am längsten halten sich die sehr feinen Rotschlammpartikel im Wasser in Schwebe. 
Die durch den Rotschlammzusatz bedingten Veränderungen der pH-Werte in den Versuchsflaschen sind in Tabelle 1 dargestellt. Diese Tabelle umfaßt die Wirkung aller, im Verlauf auch der folgenden Versuche verwendeten Rotschlammkonzentrationen. Der pH-Wert des für die Versuche verwendeten Seewassers betrug 8,05 $\pm 0,01$, der des Rotschlammes 12,21. Bei Zugabe von suspendiertem Rotschlarnm zu 1 I Seewasser machte sich eine $\mathrm{pH}$-Verschiebung erst bei Mengen von $0,05 \mathrm{~g}$ an bemerkbar. Bei dieser Konzentration stieg der Wert 2 min. nach Zugabe auf 8,12 an und sank in den folgenden 24 Stunden wieder auf 8,07 ab. Bei größeren Mengen wurde der Maximalwert erst zu einem späteren Zeitpunkt erreicht (Tab. 1), wobei der Rotschlamm durch ein Rührwerk in Turbulenz gehalten wurde.

Der Anstieg der Zelldichten in den Kulturen ist in Abbildung 1 dargestellt. In der Kontrolle stieg die Dichte innerhalb von fünf Tagen von 100 auf ca. 1400 Zellen $/ \mathrm{ml}$ an. Unter Rotschlammeinfluß zeigte sich bei 0,05 und $0,1 \mathrm{~g} / 1$ Rotschlammzusatz eine deutliche Wachstumsverzögerung der Kulturen am ersten Tage nach der Zugabe, die jedoch in den folgenden Tagen überwunden wurde und zu gleicher Teilungsintensität führte wie in der Kontrolle. Bei $0,5 \mathrm{~g} / \mathrm{l}$ sank die Zelldichte nach der Zugabe am ersten Tage sogar bis auf 12 Zellen/ml ab, erholte sich in den folgenden Tagen etwas, stagnierte dann jedoch bei ca. 60 Zellen/ml. Die Zugabe von $1 \mathrm{~g} / \mathrm{l}$ ließ alle Algen am ersten 'T'ag absterben. Die anfänglich gebildeten größeren Rotschlammaggregationen zerfielen durch das Aufschütteln wieder in kleinere Partikel (10-30 $\mu \mathrm{m}$ Durchmesser).

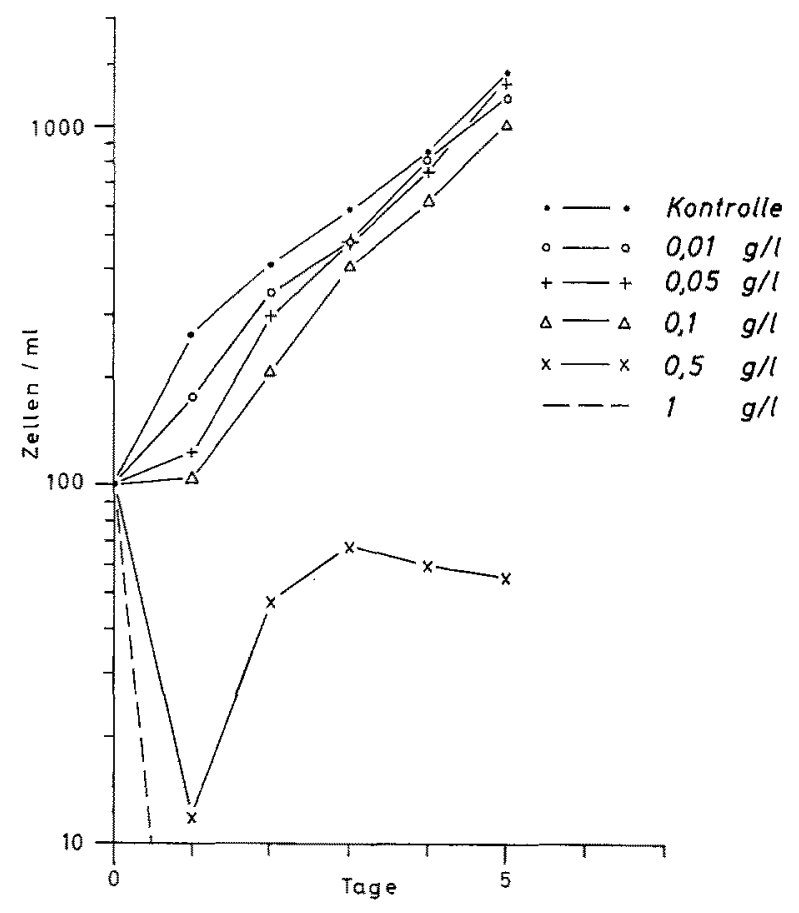

Abb. 1: Peridinium trocboideum. Batch-Kulturen nach einmaliger Zugabe von fein suspendiertem Rotschlamm bei Versuchsbeginn in Mengen von $0,01-1 \mathrm{~g} / 1$ in je 1,5 1 Schreiberlösung. Der Rotschlamm wurde täglich zweimal von Hand aufgeschüttelt (Schüttelversuch) 

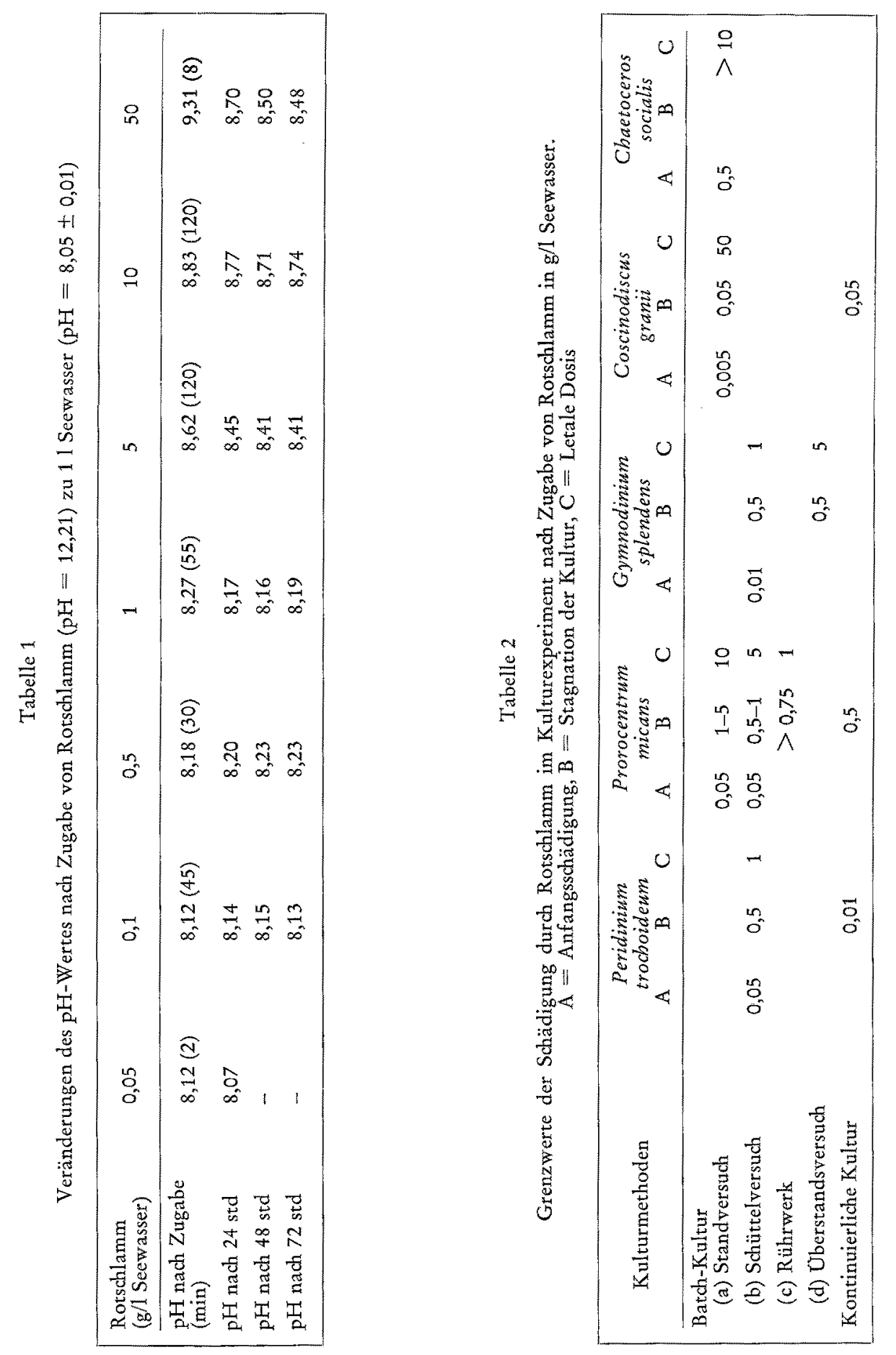
Eine erneute Zusammenballung erfolgte nicht oder nur in geringerem Maße als zu Versuchsbeginn, da die Partikel ihre anfänglich stark adhäsiven Eigenschaften weitgehend verloren hatten. Diesem Effekt ist vermutlich auch die Wiedererholung der anfangs geschädigten Algenkulturen zuzuschreiben. Frisch ins Meerwasser eingebrachter Rotschlamm beeinträchtigte die Vermehrung der Algen in weit höherem Maß als eine Suspension gealterter Rotschlammpartikel gleicher Konzentration. Der Versuch mußte nach fünf Tagen aus technischen Gründen (Ausfall eines Kühlaggregats) abgebrochen werden.

Zur Absicherung und Erweiterung des Ergebnisses wurde daher ein zweiter Ver such unter gleichen Ausgangsbedingungen angesetzt, in dem jedoch neben den Kontrollen nur die kritischen Konzentrationen von $0,1,0,5$ und $1 \mathrm{~g} / 1$ Rotschlammzusatz getestet wurden. In Abbildung 2 sind die Ergebnisse der fünffach durchgeführten Versuche dargestellt. Die Kontrolle erreichte nach elf Tagen die maximale Dichte von ca. $70000 \mathrm{Zellen} / \mathrm{ml}$. In den folgenden sieben Versuchstagen sank die Dichte auf ca.

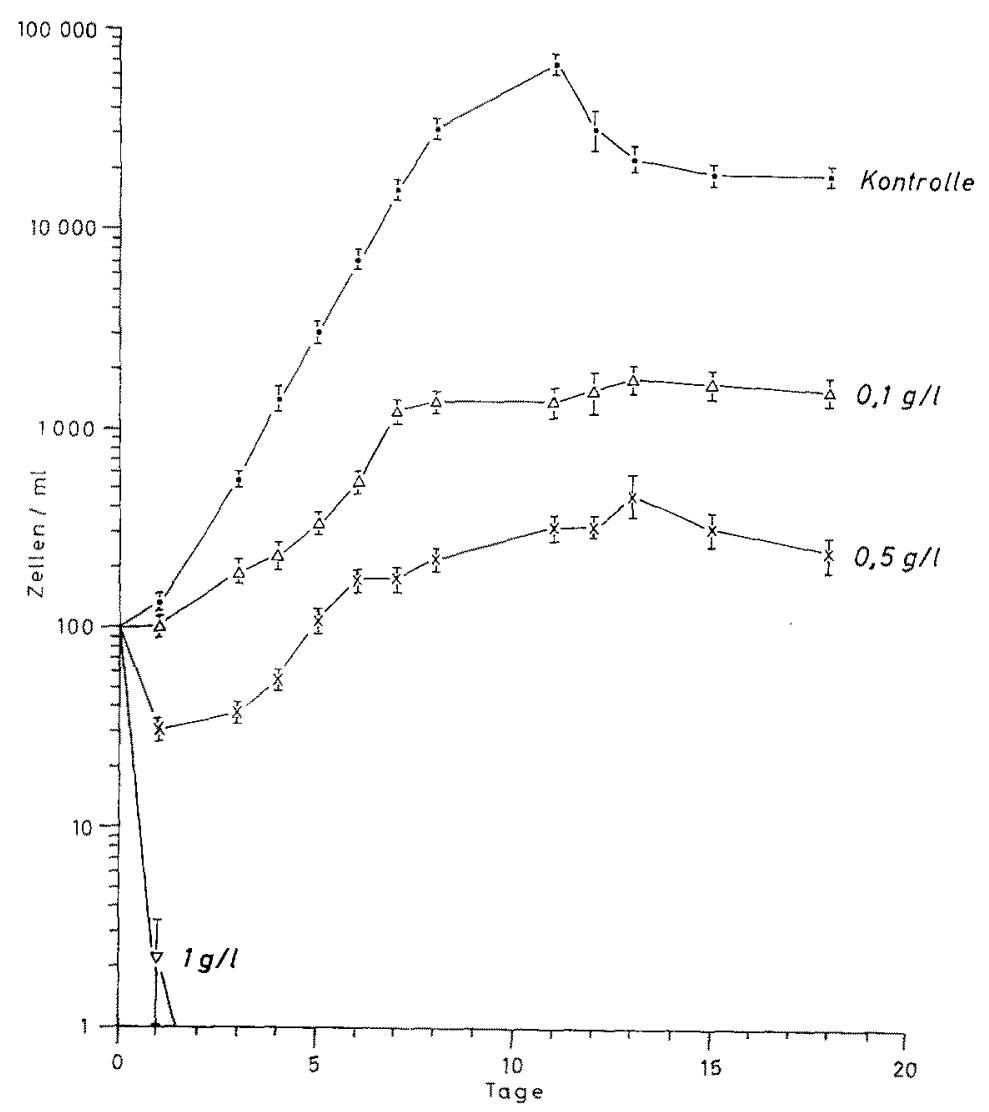

Abb. 2: Peridinium trochoideum. Batch-Kulturen nach einmaliger Zugabe von fein suspendiertem Rotschlamm bei Versuchsbeginn in Mengen von $0,1-1 \mathrm{~g} / 1$ in je 1,5 1 Schreiberlösung. Der Rotschlamm wurde täglich zweimal von Hand aufgeschüttelt. Mittelwerte und Standardabweichungen aus 5 parallelen Versuchsreihen (Schüttelversuch) 
19000 Zellen $/ \mathrm{ml}$ ab. Bei Zugabe von Rotschlamm in Mengen von 0,1 g/l blieb die Teilungsrate deutlich hinter der Kontrolle zurück und erreichte am 13. Tage nur eine Maximaldichte von ca. 1800 Zellen/ml. Bei $0,5 \mathrm{~g} / 1$ Rotschlammzusatz sank die Zelldichte einen Tag nach der Zugabe auf ca. 30 Zellen $/ \mathrm{ml}$ ab, erholte sich dann jedoch und erreichte am 13. Versuchstage die Maximaldichte von ca. 470 Zellen $/ \mathrm{ml}$. Bei $1 \mathrm{~g} / 1$ Rotschlammzusatz waren am zweiten Versuchstag alle Zellen abgestorben. Der Versuch zeigt, daß die Kulturen unter Rotschlammzusatz zwar in der Lage sind, die Anfangsschädigung bis zu einem gewissen Grade zu überwinden, dennoch aber bei weitem nicht die gleichen Zelldichten erreichen wie die Kontrollen.

\section{Kontinuierliche Kultur}

Als Ausgangsmaterial für die im Turbidostat-Prinzip betriebene kontinuierliche Kultur diente eine exponentiell wachsende Batch-Kultur. Von dieser Kultur wurde eine Serie von sechs 1-1-Gefäßen mit einer Dichte von je $1000 \mathrm{Zellen} / \mathrm{ml}$ angesetzt (vgl. p. 359). Zur Aufrechterhaltung der konstanten Zelldichte von $1000 \mathrm{Zellen} / \mathrm{ml}$ in der Kontrollkultur ergab sich ein Durchfluß des Kulturmediums von $22 \pm 3 \mathrm{ml} / \mathrm{l} / \mathrm{h}$. Rotschlamm wurde den fünf mit gleichem Durchfluß des Kulturmediums gefahrenen Testkulturen täglich einmal in Mengen von $0,01,0,05,0,1,0,5$ und $1 \mathrm{~g} / 1$ von Hand zudosiert, der Sedimentation überlassen und während der ganzen Versuchszeit nicht wieder aufgewirbelt.

In Abbildung 3 sind die Versuchsergebnisse dargestellt. Der obere Teil der Abbildung (A) zeigt die Abnahme der Zelldichten in den mit Rotschlamm versetzten Kulturen, der untere Teil (B) die durch die tägliche Zugabe bedingte Anreichernug des sedimentierten Rotschlammes am Boden der Versuchsgefäße.

Eine kontinuierliche Zugabe des Rotschlammes zusammen mit dem Nährmedium konnte nicht durchgeführt werden, da der Rotschlamm bereits in den Schlauchleitungen des Pumpensystems und im Versuchsgefäß sedimentierte. Zugleich war die Fließgeschwindigkeit auch in der Absaugleitung des Durchlaufsystems so gering, daß der Rotschlamm nicht wieder aus dem Kulturgefäß entfernt werden konnte. Die durch die tägliche Zugabe bedingte Anreicherung sedimentierten Rotschlammes am Boden der Versuchsgefäße mußte daher in Kauf genommen werden.

Bei Rotschlammzusatz von $0,01 \mathrm{~g} / / /$ Tag erfolgte in den ersten acht Tagen im Vergleich zur Kontrolle noch keine Beeinträchtigung der Teilungsintensität der Algen (Abb. 3). Dies stimmt mit früheren Ergebnissen überein (Abb. 1). Vom 9. Tag an begann die Kultur jedoch zusammenzubrechen und unterschritt am 14. Versuchstage die Dichte von 1 Zelle $/ \mathrm{ml}$. Bei Zugabe von $0,05 \mathrm{~g} / 1 / \mathrm{T} a g$ sank die Zelldichte gleich vom ersten Versuchstage an ab. Auch im Batch-Versuch (Abb. 1) hatte sich bei diesem Wert bereits eine Anfangsschädigung gezeigt, die sich im hier durchgeführten kontinuierlichen Verfahren mehr oder weniger geradlinig bis zum Absterben der Kultur fortsetzte. Das gleiche gilt für die Rotschlammzugaben in Mengen von 0,1, 0,5 und $1 \mathrm{~g} / \mathrm{l} /$ Tag. Tägliche Zugabe frischen Rotschlammes im kontinuierlichen Verfahren summiert demnach die im Batch-Verfahren beobachtete Anfangsschädigung der Kulturen und zeigt, daß eine über längere Zeit fortgesetzte „Dauerberegnung“ mit frischem Rot- 
schlamm sogar in nicht anfangsschädigender Menge zum Absterben der Kultur führt $(0,01 \mathrm{~g} / 1 / \mathrm{Tag})$.

Die in Abbildung 3A eingetragene gestrichelte Gerade gibt die durch den Wasseraustausch bedingte Verdünnung der Kulturen an. Verlaufen die Kurven der Zelldichten in den Kulturen zu dieser Geraden parallel, so stagniert die Vermehrung der
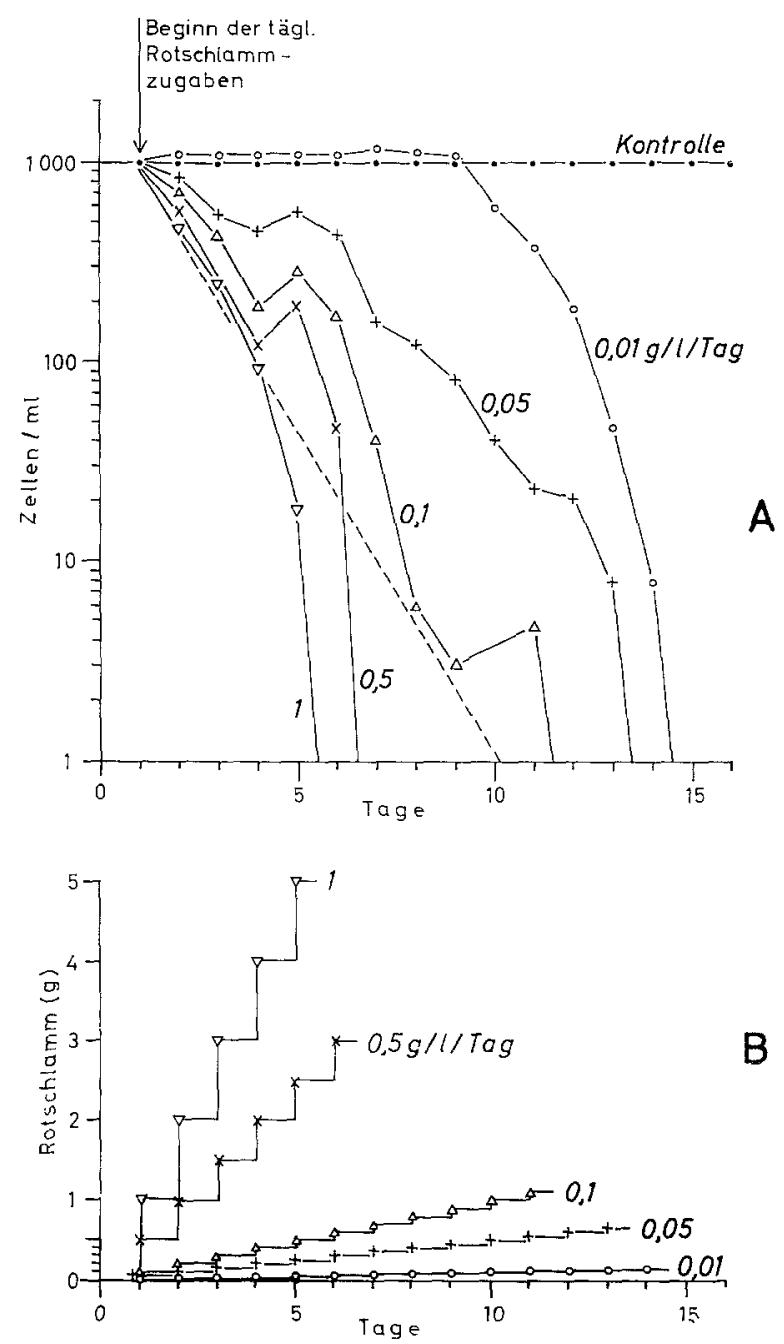

Abb. 3: Peridinium trocboideum. Kontinuierliche Kulturen (Turbidostate) mit täglicher $\mathrm{Zu}$ dosierung von frischem, fein suspendiertem Rotschlamm in Mengen von $0,01-1 \mathrm{~g} / 1 / \mathrm{Tag}$ in 1-1-Gefäßen mit Schreiberlösung. Der Rotschlamm wurde nach seiner Sedimentation nicht wieder aufgewirbelt. (A) Abfall der Zelldichten unter Rotschlammeinfluß (als gestrichelte Linie ist die durch den kontinuierlichen Durchfluß des Kulturmediums bedingte theoretische Verdünnung der Kultur angegeben). (B) Zunahme des sedimentierten Rotschlammes am Boden der Versuchsgefäße 
Algen; unterschreitet der Abfall der Zellkurven den Anfall der Geraden, so sterben die Kulturen ab. Im Falle der Zugabe von $0,01 \mathrm{~g} / 1 /$ Tag wurde dieser Zustand in den drei letzten Versuchstagen bereits erreicht.

\section{Prorocentrum micans}

\section{Batch-Kulturen}

(a) St andversuch. Die Algen wurden in neun 1,5-I-Gefäßen mit einer Anfangszelldichte von je $50 \mathrm{Zellen} / \mathrm{ml}$ in exponentieller Wachstumsphase angesetzt. Rotschlamm wurde in acht Konzentrationsstufen in Mengen von 0,005 bis $10 \mathrm{~g} / 1$ einmalig bei Versuchsbeginn zugesetzt, der Sedimentation überlassen und nicht wieder aufgewirbelt (vgl. p. 359). Die Algen hatten daher die Gelegenheit, sich nach der Sedimentation des Schlammes im überstehenden Nährmedium frei zu entwickeln.

Die Kontrolle wuchs in 16 Tagen bis zur Dichte von ca. $8000 \mathrm{Zellen} / \mathrm{ml}$ heran (Abb. 4). Von $0,05 \mathrm{~g} / 1$ Rotschlammzugabe an wurde in den ersten Tagen des Versuchs

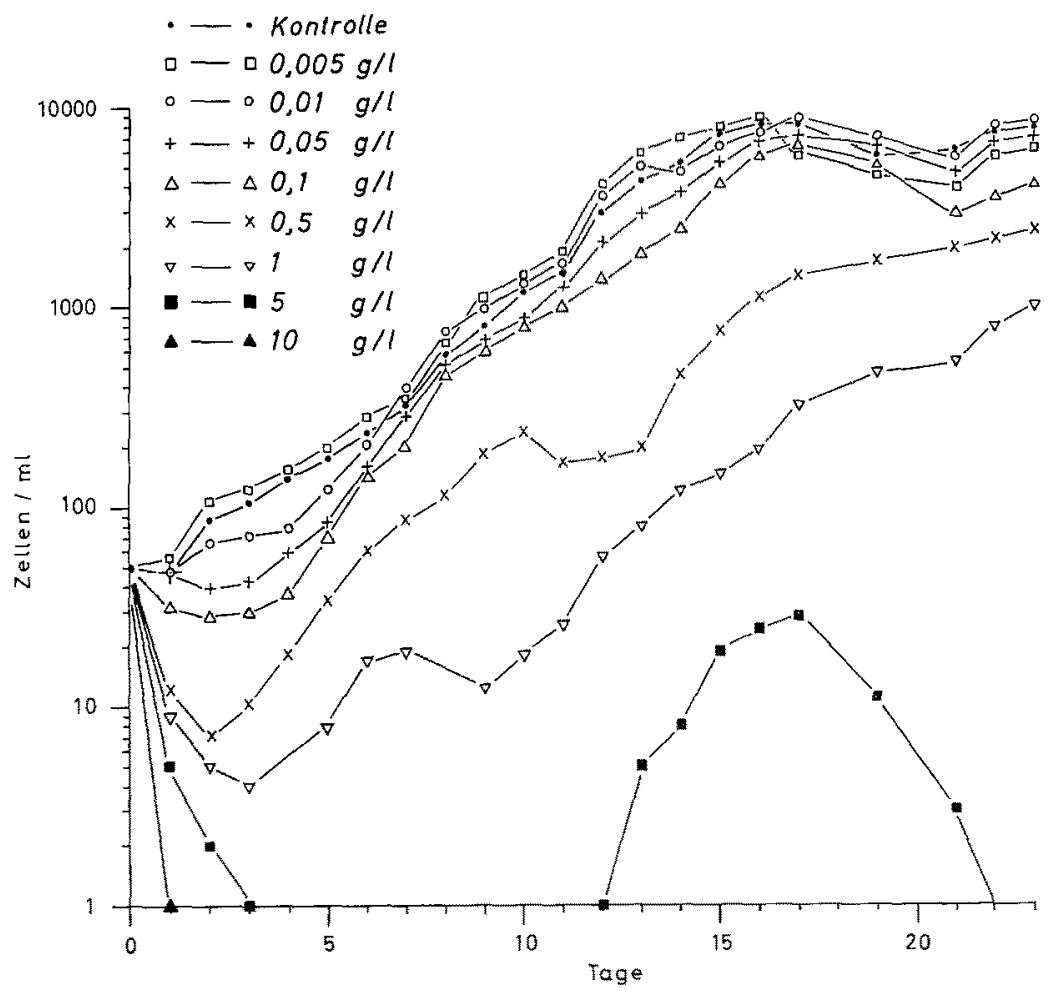

Abb. 4: Prorocentrum micans. Batch-Kulturen nach einmaliger Zugabe von fein suspendiertem Rotschlamm bei Versuchsbeginn in Mengen von $0,005-10 \mathrm{~g} / \mathrm{in}$ je 1,5 1 Schreiberlösung. Der Rotschlamm wurde nach seiner Sedimentation nicht wieder aufgewirbelt (Standversuch) 
eine Anfangsschädigung deutlich, die aber bis zur Konzentration von $0,1 \mathrm{~g} / 1$ ausgeglichen wurde und bei Versuchsende zu annähernd gleichen maximalen Zelldichten führte wie in der Kontrollkultur. In den Konzentrationen von 0,5 und $1 \mathrm{~g} / 1$ blieben die Kulturen stark hinter der Kontrolle zurück. Die Anfangsschädigung drückte die Zellzahl auf sieben bzw. vier Zellen/ml herunter. Bei Versuchsende am 23. Tage wurden erst Dichten von $2500 \mathrm{bzw} .1000 \mathrm{Zellen} / \mathrm{ml}$ erreicht. Bei $5 \mathrm{mg} / \mathrm{l}$ Rotschlammzugabe sank die Zelldichte nach drei Tagen auf Werte unter $1 \mathrm{Zelle} / \mathrm{ml}$ ab. Erst am zwölften Versuchstage konnten die Zellen wieder im Versuch nachgewiesen werden, erreichten an 17. Versuchstage eine Dichte von knapp $30 \mathrm{Zellen} / \mathrm{ml}$ und starben dann wieder ab. Nach Zugabe von $10 \mathrm{~g} / 1$ sank die Zelldichte bereits nach einem Tage auf den Wert von $1 \mathrm{Zelle} / \mathrm{ml}$. Die Kultur starb dann ganz ab.

In einem anderen Experiment (KAYSER, im Druck) wurde der Rotschlamm bei sonst gleicher Versuchsanordnung in Mengen von 5, 10 und $50 \mathrm{~g} / \mathrm{l}$ unzerteilt in Form eines Brockens zugegeben. Dieser Brocken löste sich nicht auf, sondern blieb am Boden des Gefäßes liegen. Die Beeinflussung der Kulturen war weit geringer als bei Zugabe gleicher Mengen in fein suspendierter Form. Es konnte lediglich eine Anfangsschädigung registriert werden, die nach ca. fünf Tagen überwunden wurde und in ein exponentielles Wachstum der Kulturen überging.

(b) Schüttelversuch. Die Algen wurden wiederum in neun 1,5-l-Gefäßen

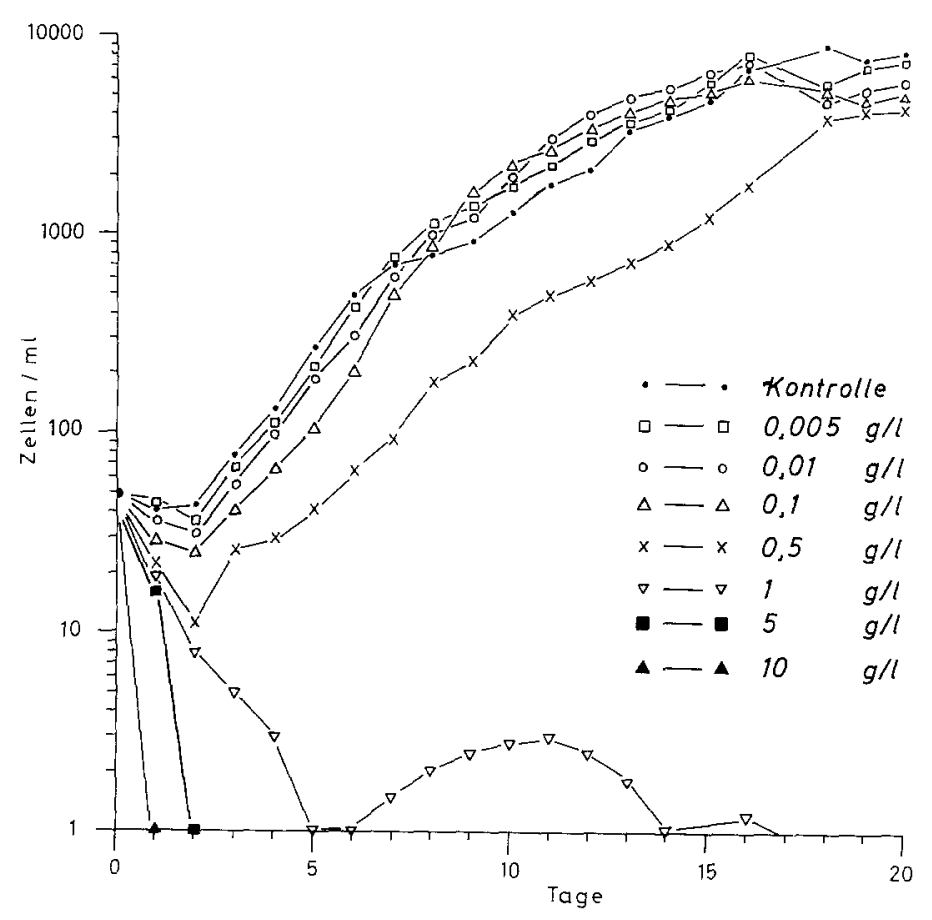

Abb. 5: Prorocentrum micans. Batch-Kulturen nach einmaliger Zugabe von fein suspendiertem Rotschlamm bei Versuchsbeginn in Mengen von $0,005-10 \mathrm{~g} / 1$ in je 1,5 1 Schreiberlösung. Der Rotschlamm wurde täglich zweimal von Hand aufgeschüttelt (Schüttelversuch) 
mit einer Anfangsdichte von je 50 Zellen/ml angesetzt. Der in acht Konzentrationsstufen bei Versuchsbeginn einmalig zugegebene Rotschlamm $(0,005$ bis $10 \mathrm{~g} / \mathrm{l})$ wurde dagegen täglich morgens und abends von Hand aufgeschüttelt (vgl. p. 359). Die Algen waren daher zweimal am Tage der Sedimentationswirkung der Rotschlammpartikel ausgesetzt. Abbildung 5 zeigt die Ergebnisse. Bis zur Konzentration von 0,5 g/1 Rotschlammzugabe entsprach der Wachstumsverlauf der Kulturen etwa dem des Standversuchs (Abb. 4). Nach Zugabe von $1 \mathrm{~g} / 1$ sank die Zelldichte jedoch nach fünf Tagen bis auf $1 \mathrm{Zelle} / \mathrm{ml} \mathrm{ab}$ und konnte sich bis zum elften Tage nur unwesentlich bis auf 3 Zellen/ml erholen. Anschließend starb die Kultur gänzlich ab. 5 und $10 \mathrm{~g} / 1$ Rotschlammzugabe führte zum schnellen Ende der Kulturen nach ein bzw. zwei Tagen.

In beiden Experimenten wird wiederum die starke Anfangsschädigung der Kulturen unmittelbar nach der Zugabe des frischen Rotschlammes deutlich. Ein Vergleich der beiden Versuche zeigt, daß sich ein ständig wiederholtes Aufschütteln des Rotschlammes zusätzlich negativ auf die Kulturen auswirkte. Daß der Effekt nicht größer war, ist dem Umstand zuzuschreiben, daß die sedimentierenden Rotschlammpartikel im Verlaufe des Versuchs gealtert waren und daher ihre adhäsiven Wirkungen weitgehend verloren hatten. Das ständig wiederholte Aufschütteln des Schlammes bewirkte

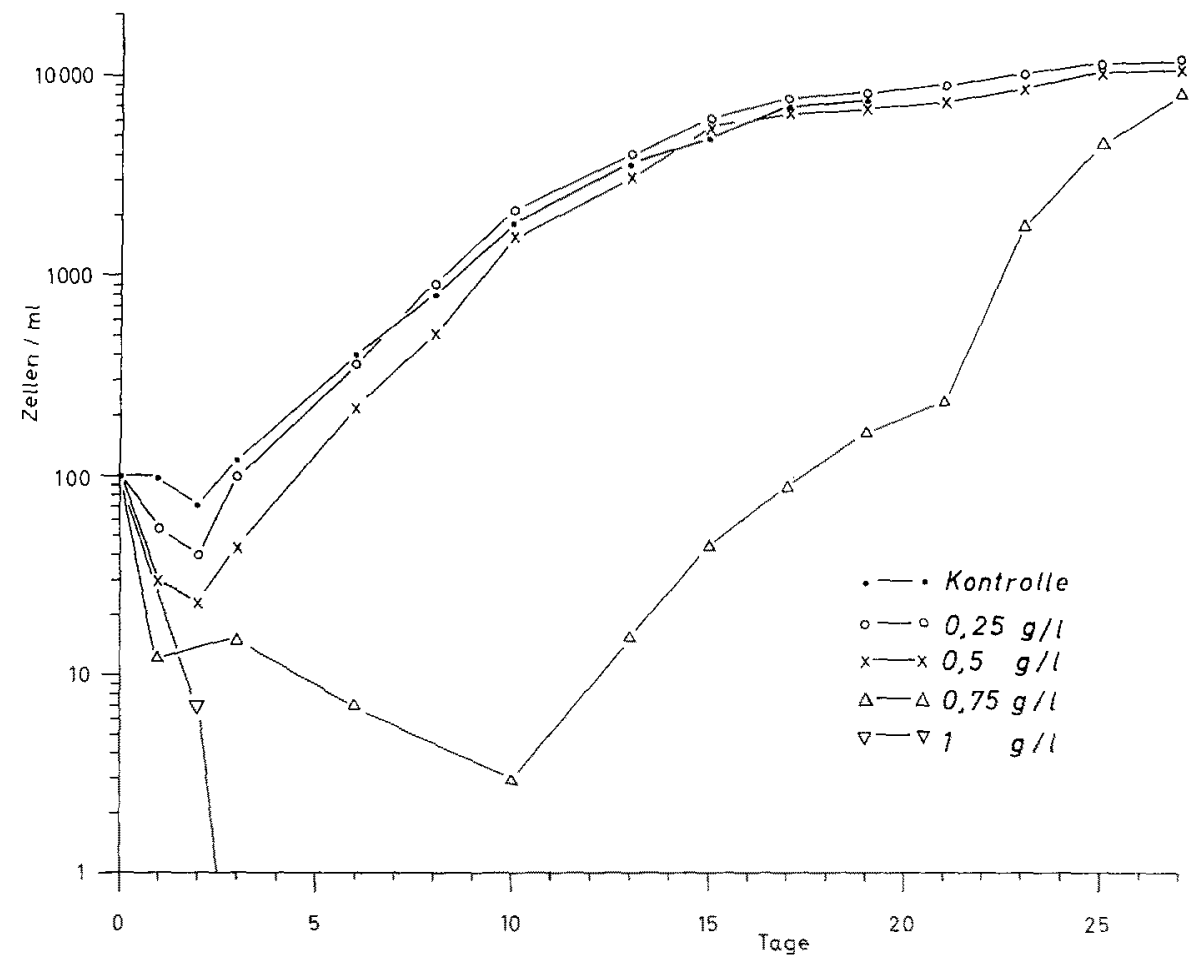

Abb. 6: Prorocentrum micans. Batch-Kulturen nach einmaliger Zugabe von fein suspendiertem Rotschlamm bei Versuchsbeginn in Mengen von $0,25-1$ g/l in je 51 Schreiberlösung. Der Rotschlamm wurde während der Versuchszeit durch Rührwerke soweit wie möglich kontinuierlich in Suspension gehalten (ca. $200 \mathrm{U} / \mathrm{min}$ ) (Rührwetksversuch) 
auch eine zeitweise stark erhöhte Trübung des Versuchswassers, die sich auf den Lichteinfall und damit negativ auf die Kulturen auswirkte.

(c) R ührwerksversuch. Die Algen wurden in fünf 5-1-Gefäßen mit einer Anfangsdichte von $100 \mathrm{Zellen} / \mathrm{ml}$ angesetzt. Rotschlamm wurde einmalig bei Versuchsbeginn in Mengen von 0,25, 0,5, 0,75 und $1 \mathrm{~g} / \mathrm{l}$ zugesetzt und so weit wie möglich durch Rührwerke (ca. $200 \mathrm{U} / \mathrm{min}$ ) kontinuierlich in Turbulenz gehalten (vgl. p. 359). Die Rührgeschwindigkeit reichte nicht aus, um den Rotschlamm quantitativ in Schwebe zu halten, dennoch wurde der größte Teil des Schlammes aufgewirbelt. Höhere Rührgeschwindigkeiten konnten nicht erzeugt werden, weil sonst das Wachstum der Algen auch in der Kontrolle negativ beeinflußt worden wäre. Die Kontrolle unterlag zunächst einer Lag-Phase von zwei Tagen, wuchs bis zum 19. Tag dann auf die Dichte von ca. $8000 \mathrm{Zellen} / \mathrm{ml}$ heran (Abb. 6). Unter Rotschlammzusatz reagierten die Kulturen in allen verwendeten Konzentrationen mit deutlicher Anfangsschädigung. Bei 0,75 g/1 sank die Zelldichte bis zum zehnten Tage auf $3 \mathrm{Zellen} / \mathrm{ml} \mathrm{ab}$, erholte sich dann jedoch. Bei $1 \mathrm{~g} / 1$ starb die Kultur nach drei Tagen ab.

Ein Vergleich der drei Experimente zeigt, daß sich die schädliche Wirkung des Rotschlammes erhöht, je mehr Partikel während des Versuchs in Schwebe waren. Im ersten Versuch gelang es den Algen, sich noch über einer Konzentration von $5 \mathrm{~g} / 1$ überwiegend sedimentierten Rotschlammes zeitweilig zu vermehren. Im zweiten Versuch war diese Grenze auf $1 \mathrm{~g} / 1$ heruntergedrückt. Im dritten Versuch starben die Algen bei $1 \mathrm{~g} / 1$ Rotschlammzusatz sofort ab.

(d) Überstandsversuch. Die Algen wurden mit einer Anfangsdichte von

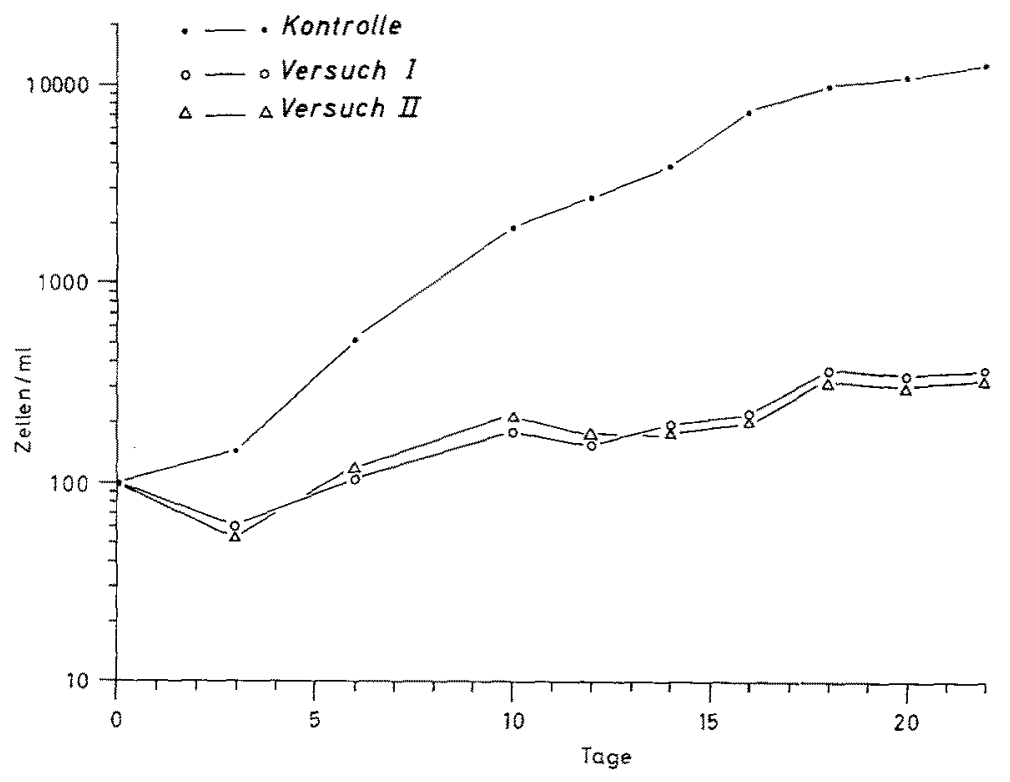

Abb. 7: Prorocentrum micans. Batch-Kulturen. Kontrolle in Schreiberlösung. Für Versuche $I$ und II wurden die Algen in die abdekantierte Oberstandslösung einer mit $250 \mathrm{~g}$ Rotschlamm in 11 Seewasser angesetzten und über einen Tag in ruhender Wassersäule sedimentierten Rotschlammprobe eingesetzt (Überstandsversuch) 
$100 \mathrm{Zellen} / \mathrm{ml}$ in der abdekantierten Uberstandslösung einer über einen Tag sedimentierten Rotschlammprobe von $250 \mathrm{~g} / 1$ Seewasser gezüchtet (vgl. p. 359). Der pH-Wert der Uberstandslösung betrug 9,26. Abbildung 7 zeigt die Ergebnisse des doppelt gefahrenen Versuchs. Die Kontrolle in Schreiberlösung wuchs in 22 Tagen auf die Dichte von ca. 13000 Zellen/ml heran. In der Uberstandslösung erfolgte nur zögerndes Wachstum. Die Dichte der beiden Kulturen erreichte bei Versuchsende nur 350 und 370 Zellen $/ \mathrm{ml}$. Der Versuch zeigt, daß Prorocentrum micans auf $\mathrm{pH}-\mathrm{Veränderungen} \mathrm{und} \mathrm{die}$ aus dem Rotschlamm löslichen Produkte offenbar äußerst robust reagiert. Andererseits macht der Versuch deutlich, daß die Schädigung der Algen bei niedrigeren Rotschlammkonzentrationen in erster Linie auf die mechanische Wirkung der sedimentierenden Partikel zurïckzuführen ist, denn eine Zugabe von $10 \mathrm{~g} / 1$ Rotschlamm direkt zu den Algen wirkte bereits letal.

\section{Kontinuierliche Kultur}

Die im Turbidostat-Prinzip betriebene kontinuierliche Kultur mit Prorocentrum micans wurde in einem 8-1-Fermenter gefahren. Dieses Gerät verfügte nur über ein Kulturgefäß (vgl. p. 359). Die Algen wurden zunächst mit einer Dichte von 200 Zellen/ $\mathrm{ml}$ in Schreiberlösung in das Versuchsgefäß eingesetzt (Abb. 8A). Im Verlauf einer dreitägigen Batch-Phase wurden sie in exponentiellem Wachstum bis auf die Dichte von ca. 650 Zellen/ml herangezüchtet. Dann wurde der Durchlauf eingeschaltet, der ein Abfangen der exponentiell wachsenden Kultur auf annähernd konstanter Zelldichte bewirkte. Die der Vermehrungsrate der Algen entsprechende Pumpgeschwindigkeit betrug $370 \mathrm{ml} / \mathrm{l} / \mathrm{Tag}$. Vom zehnten Versuchstage an wurde der Kultur dann täglich einmal frischer Rotschlamm in fein suspendierter Form von Hand zudosiert. Die Dosis betrug am ersten Tage der Zugabe $0,1 \mathrm{~g} / 1$. Diese Menge verteilte sich auf Grund der durch Belüftung hervorgerufenen Turbulenz sofort im ganzen Gefäß und begann dann zu sedimentieren. Der Bodensatz wurde während des ganzen Versuches nicht wieder aufgewirbelt. Die Zelldichte der Kultur war nach dieser Dosis am folgenden Tage auf den Wert von 520 Zellen/ml abgesunken. Da zunächst die kritische Grenzkonzentration einer ersten, gerade meßbaren Schädigung ermittelt werden sollte, wurde die zugegebene Rotschlammenge auf $0,03 \mathrm{~g} / \mathrm{l} / \mathrm{Tag}$ reduziert. Im Verlauf von 15 Tagen pendelte sich die Zelldichte bei dieser Dosis nach anfänglicher Zunahme auf einen relativ konstanten Wert von ca. $500 \mathrm{Zellen} / \mathrm{ml}$ ein. Die Rotschlammzugabe wurde daher am 25. Versuchstage wieder erhöht auf die Menge von 0,1 g/l/Tag. Auch hier war nach zwei Tagen noch keine negative Reaktion der Algen meßbar. Die Zellzahl nahm vielmehr im Rahmen der leichten Schwankungen der vorhergehenden Tage auf 550 Zellen/ml zu. Die Rotschlammdosis wurde daher am 27. Versuchstage nochmals drastisch auf $0,5 \mathrm{~g} / \mathrm{l} / \mathrm{Tag}$ erhöht. Nun zeigte sich ein rapides Absinken der Zelldichte, die innerhalb weniger Tage zum Absterben der Kultur führte. In Abbildung $8 \mathrm{~B}$ ist die Menge des zugegebenen und zum überwiegenden Teil am Boden des Versuchsgefäßes sedimentierten Rotschlammes angegeben.

Der Versuch zeigt, daß die kritische erste Beeinflussung der Vermehrungsrate von Prorocentrum micans bei täglicher Zugabe von frischem Rotschlamm zwischen einer Dosis von 0,1 und 0,5 g/l/Tag liegt. Dieser Wert entspricht den Ergebnissen der Batch- 
Versuche, bei denen sich die Anfangsschädigung unmittelbar nach der Zugabe des frischen Rotschlammes in den gleichen Konzentrationen bereits deutlich bemerkbar machte. Im kontinuierlichen Versuch summierte sich diese Schädigung bis zum Absterben der Kultur, ohne daß es zu einer Erholungsphase kommen konnte.

\section{Gymnodinium splendens}

\section{Batch-Kulturen}

(a) $\mathrm{S} c h$ ü $t \mathrm{tel}$ ver such. Die Algen wurden in acht 1,5-1-Gefäßen mit einer Anfangsdichte von je 100 Zellen/ml angesetzt. Rotschlamm wurde den Kulturen in sieben Konzentrationsstufen in Mengen von 0,01 bis $10 \mathrm{~g} / 1$ bei Versuchsbeginn einmalig zugegeben und täglich zweimal aufgeschüttelt (vgl. p. 359). Die Kontrolle wuchs
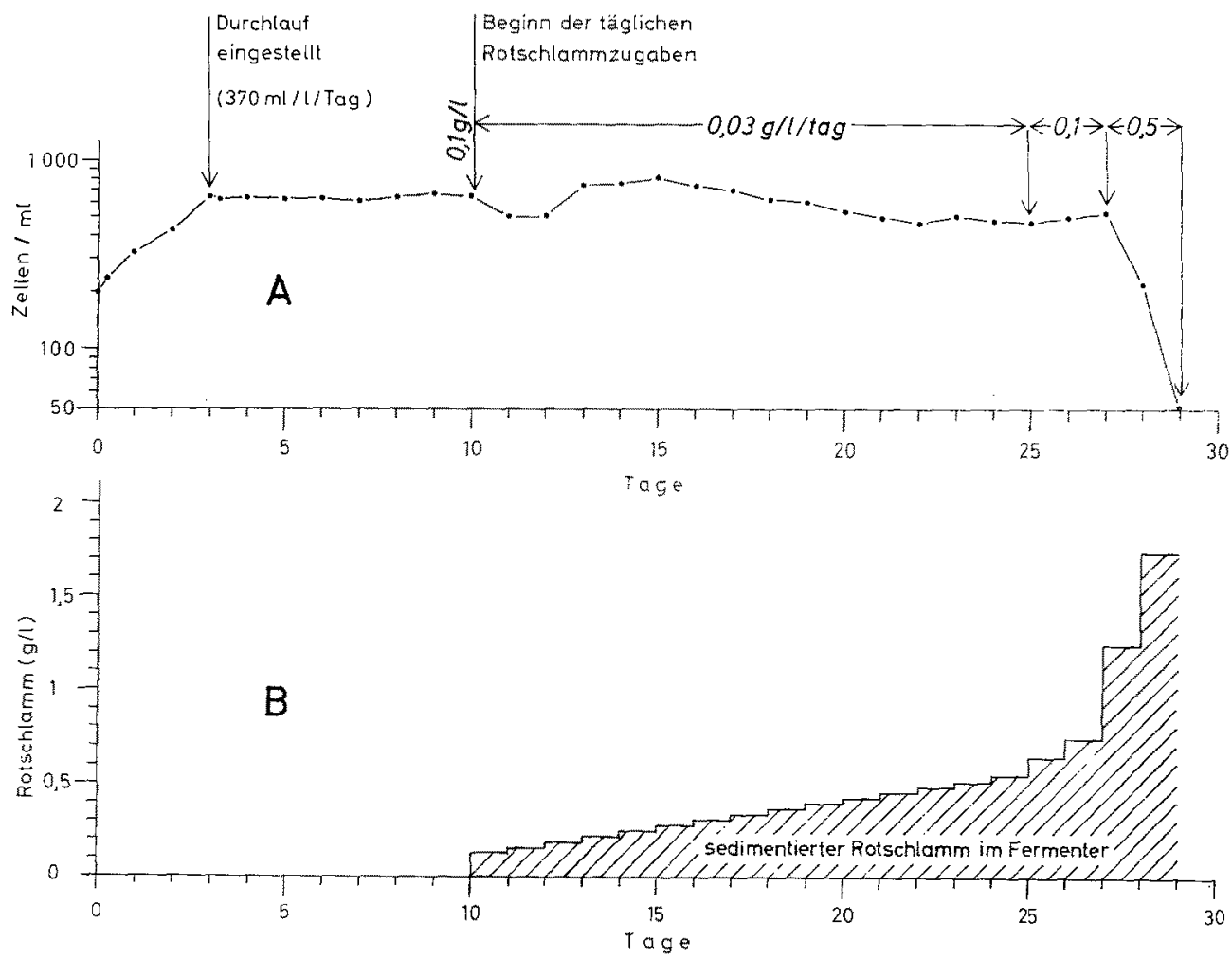

Abb. 8: Prorocentrum micans. Kontinuierliche Kultur (Turbidostat) mit täglicher Zudosierung von frischem, fein suspendiertem Rotschlamm in Mengen von 0,03-0,5 g/V/Tag in einem 8-1Fermenter in Schreiberlösung. Der Rotschlamm wurde nach seiner Sedimentation nicht wieder aufgewirbelt. (A) Zelldichte der Kultur in Abhängigkeit vom Durchlauf des Kulturmediums und von den Rotschlammzugaben. (B) Zunahme des sedimentierten Rotschlamms am Boden des Versuchsgefäßes 
in fünf Tagen auf die Dichte von ca. 630 Zellen/ml heran (Abb. 9). Der Versuch mußte zu diesem Zeitpunkt durch Ausfall eines Kühlaggregates abgebrochen werden. In den mit Rotschlamm versetzten Kulturen zeigte sich nach Zugabe von $0,01-0,1 \mathrm{~g} / \mathrm{l}$ bereits eine deutliche und mit der Konzentration steigende Anfangsschädigung einen Tag nach Versuchsbeginn. Trotz täglicher Aufschüttelung des Rotschlammes erholten sich die Kulturen jedoch anschließend und wuchsen über vier Tage mit annähernd gleicher Teilungsintensität wie die Kontrolle. Nach Zugabe von $0,5 \mathrm{~g} / 1$ sank die Dichte nach einem Tag von 100 auf $50 \mathrm{Zellen} / \mathrm{ml} \mathrm{ab}$, stagnierte auf dieser Dichte und zeigte am fünften Tage abermals eine fallende Tendenz. Nach Zugabe von $1 \mathrm{~g} / 1$ sank die Zelldichte nach vier Tagen, nach Zugabe von $5 \mathrm{~g} / 1$ nach einem Tag auf Werte von weniger als $10 \mathrm{Zellen} / \mathrm{ml} \mathrm{ab}$, ohne sich wieder zu erholen. Nach Zugabe von $10 \mathrm{~g} / \mathrm{l}$ konnten bereits nach einem Tage keine Zellen mehr nachgewiesen werden.

(b) Überstandsversuch. In diesem Experiment wurde die Frage untersucht, wie sich die Algen über einer Schicht sedimentierten Rotschlammes im freien Überstandswasser entwickeln können, ohne an der Sedimentation teilgenommen zu haben. Hierfür wurde Rotschlamm in fein suspendierter Form in vier Konzentrationsstufen in Mengen von $0,5-10 \mathrm{~g} / \mathrm{l}$ in 5-1-Gefäße eingebracht. Nach anfänglicher guter Durchmischung wurde der Schlamm über zwei Tage der Sedimentation überlassen und lediglich die bei allen Versuchen verwendete schwache Durchlifftung beibehalten. Dann erst wurden die Algen mit einer Anfangsdichte von $100 \mathrm{Zellen} / \mathrm{ml}$ vorsichtig eingesetzt, möglichst ohne den Bodensatz aufzurühren (vgl. p. 359). Die Kontrolle wuchs in 19 Tagen auf die Dichte von ca. 5000 Zellen/ml heran (Abb. 10). Bis zum achten Ver-

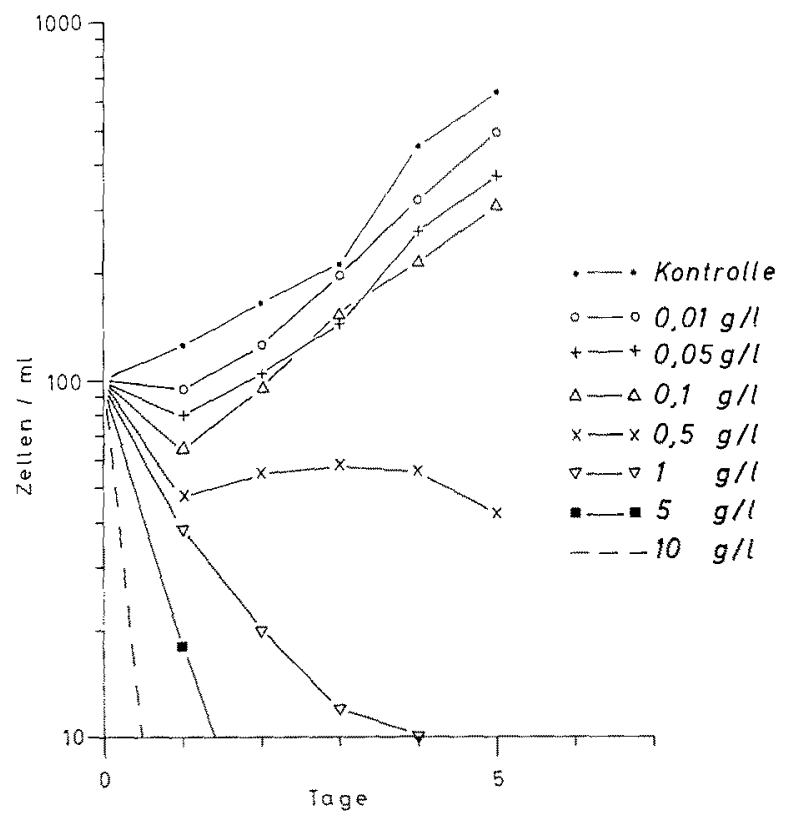

Abb. 9: Gymnodinium splendens. Batch-Kulturen nach einmaliger Zugabe von fein suspendiertem Rotschlamm bei Versuchsbeginn in Mengen von $0,01-10 \mathrm{~g} / 1$ in je 1,51 Schreiberlösung. Der Rotschlamm wurde täglich zweimal von Hand aufgeschüttelt (Schüttelversuch) 
suchstage folgten ihr die mit Rotschlamm versetzten Kulturen mit mehr oder weniger gleicher Teilungsintensität. Dann jedoch begannen die Algen über 0,5 und $1 \mathrm{~g} / 1$ Rotschlammsediment auf annähernd gleichen Zelldichten zu stagnieren. Über $5 \mathrm{~g} / \mathrm{l}$ Rotschlamm sank die Kultur noch vor Versuchsende auf die Dichte von weniger als $10 \mathrm{Zellen} / \mathrm{ml} \mathrm{ab}$ und ging ein. Uber $10 \mathrm{~g} / 1$ zeigten die Zellen nach dem zehnten Versuchstage atypische Erscheinungsbilder und starben im Verlaufe eines weiteren Tages ab. Die atypischen Zellen waren kleiner und unregelmäßig deformiert.

Ein Vergleich der beiden Versuche zeigt, daß die Algen bei direktem Kontakt mit frischem, sedimentierendem Rotschlamm bereits nach. Zugabe von $0,01 \mathrm{~g} / 1$ mit einer Anfangsschädigung reagierten und bei $0,5 \mathrm{~g} / 1$ Rotschlammzusatz in eine Phase der Stagnation übergingen (Schüttelversuch). Aber auch ohne direkten Kontakt mit dem sedimentierenden Rotschlamm (Überstandsversuch) trat ab 0,5 g/1 Rotschlammzugabe eine Beeinflussung offenbar toxischer Natur auf, die zur Stagnation der Vermehrung der Kulturen führte. Diese Wirkung setzte über dem sedimentierten Rotschlamm erst nach acht Tagen deutlich ein und führte in den höheren Konzentrationen (5 und $10 \mathrm{~g} / \mathrm{l})$ zum Absterben unter Bildung von Zelldeformationen.

\section{Coscinodiscus granii}

\section{Batch-Kultur}

(a) St a ndvers uch. Die Algen wurden in einer Serie von zehn 2-1-Gefäßen mit einer Anfangsdichte von 100 Zellen $/ 100 \mathrm{ml}$ angesetzt. Rotschlamm wurde in neun

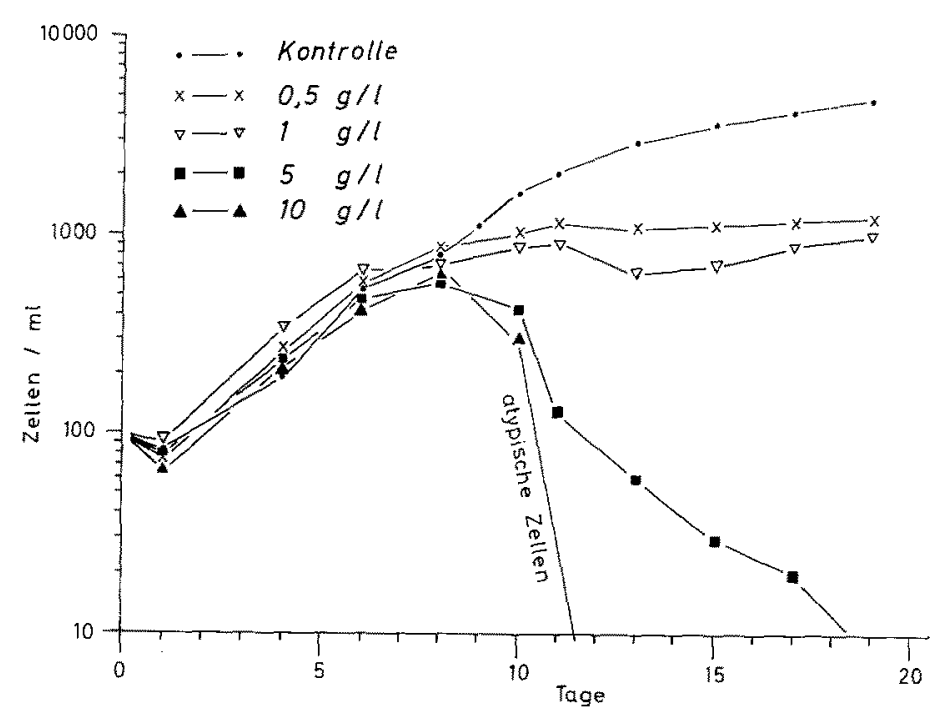

Abb. 10: Gymnodinium splendens. Batch-Kulturen in der Uberstandslösung über sedimentiertem Rotschlamm. Rotschlamm wurde in Mengen von $0,5-10 \mathrm{~g} / 1$ in je 51 Schreiberlösung eingebracht. Erst nach zweitägiger Sedimentation wurden die Algen in den Versuch eingesetzt (C̈berstandsversuch) 
Konzentrationsstufen in Mengen von 0,005 bis $50 \mathrm{~g} / 1$ einmalig bei Versuchsbeginn zugesetzt, der Sedimentation ïberlassen und nicht wieder aufgewirbelt (vgl. p. 359). Abbildung 11 zeigt den Verlauf des Versuchs nach Zugabe von 0,005-0,5 g/1 Rotschlamm. Die Kontrolle wuchs nach einer Lag-Phase yon einem Tag innerhalb weiterer vier Tage bis zur Dichte von ca. 3500 Zellen/100 ml heran. Dann endete die exponentielle Wachstumsphase der Kultur und ging in die stationäre Phase über, in der sich die Zelldichte auf Werten um 5000 Zellen $/ 100 \mathrm{ml}$ bis zum Versuchsende nach 22 Tagen etwa konstant hielt. Nach 0,005 und $0,01 \mathrm{~g} / 1$ Rotschlammzugabe zeigte sich am ersten Versuchstage eine konzentrationsabhängige Anfangsschädigung, die die Dichte der Kulturen auf.ca. $60 \mathrm{bzw} .30$ Zellen $/ 100 \mathrm{ml}$ herabdrückte. Die Kulturen erholten sich. jedoch zu gleicher Teilungsintensität und Dichte, wie in der Kontrolle. Bei $0,05-0,5$ g/1 Rotschlammzusatz fiel die Zelldichte nach einem Tage auf Werte unter 10 Zellen/100 ml ab. Die Kulturen konnten sich jedoch nicht erholen, sondern stagnierten auf dieser geringen Zelldichte mit Schwankungen zwischen 4 und $40 \mathrm{Zellen} / 100 \mathrm{ml}$. Auch nach Zugabe von 1,5 und $10 \mathrm{~g} / 1$ Rotschlamm überlebten immer noch einige Zellen bis zum Versuchsende. Erst bei $50 \mathrm{~g} / \mathrm{l}$ war die Kultur am ersten Versuchstage gänzlich abgestorben. Über erste Ergebnisse dieses Versuchs wurde bereits in anderem Zusammenhang berichtet (KAYSER, im Druck).

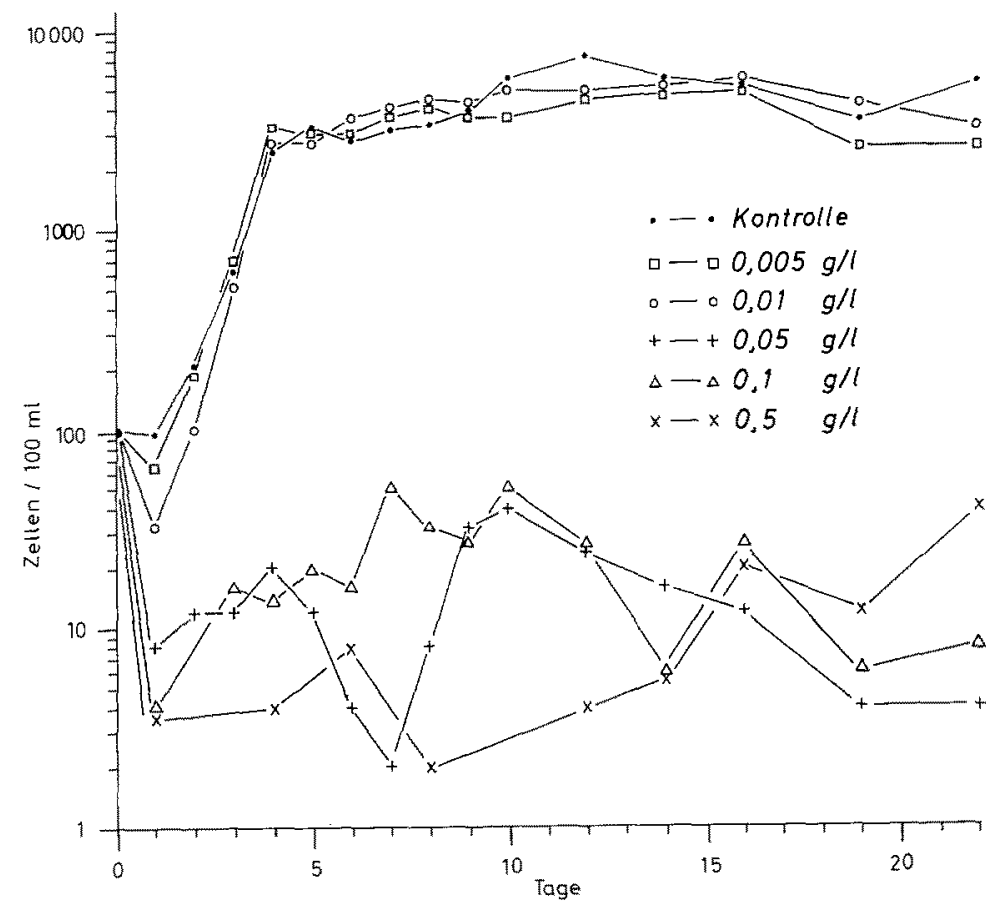

Abb. 11: Coscinodiscus granii. Batch-Kulturen nach einmaliger Zugabe von fein suspendiertem Rotschlamm bei Versuchsbeginn in Mengen von $0,005-0,5 \mathrm{~g} / 1$ in je 21 reinem Seewasser. Der Rotschlamm wurde nadh seiner Sedimentation nicht wieder aufgewirbelt (Standversuch) 


\section{Kontinuierliche Kultur}

Zur Absicherung und Erweiterung der Ergebnisse der Batch-Kultur wurde eine kontinuierliche Kultur im Turbidostatprinzip in einem 8-1-Fermenter durchgeführt (vgl. p. 359). Die Algen wurden mit einer Anfangsdichte von 100 Zellen/100 ml eingebracht (Abb. 12A). In exponentieller Phase wurden diese Zellen zunächst als BatchKultur innerhalb von vier Tagen bis zur Dichte von ca. 400/Zellen/100 ml herangezüchtet. Dann wurde das Durchlaufsystem eingeschaltet. Die Durchlaufquote betrug $180 \mathrm{ml} / \mathrm{l} / \mathrm{Tag}$ und hielt damit die exponentiell weiterwachsende Kultur auf gleicher Zelldichte. Vier Tage nach Einschalten des Durchlaufs wurde dem Kulturgefäß zum erstenmal Rotschlamm in fein suspendierter Form von Hand zudosiert. Die Menge betrug zunächst $0,01 \mathrm{~g} / 1 / \mathrm{Tag}$. Diese Dosis wurde über drei Tage lang beibehalten. Da sich an der Zelldichte der Kultur noch kein deutlich negativer Effekt bemerkbar machte, wurde die Rotschlammzugabe für weitere drei Tage auf $0,05 \mathrm{~g} / 1 / \mathrm{T}$ ag erhöht. Die
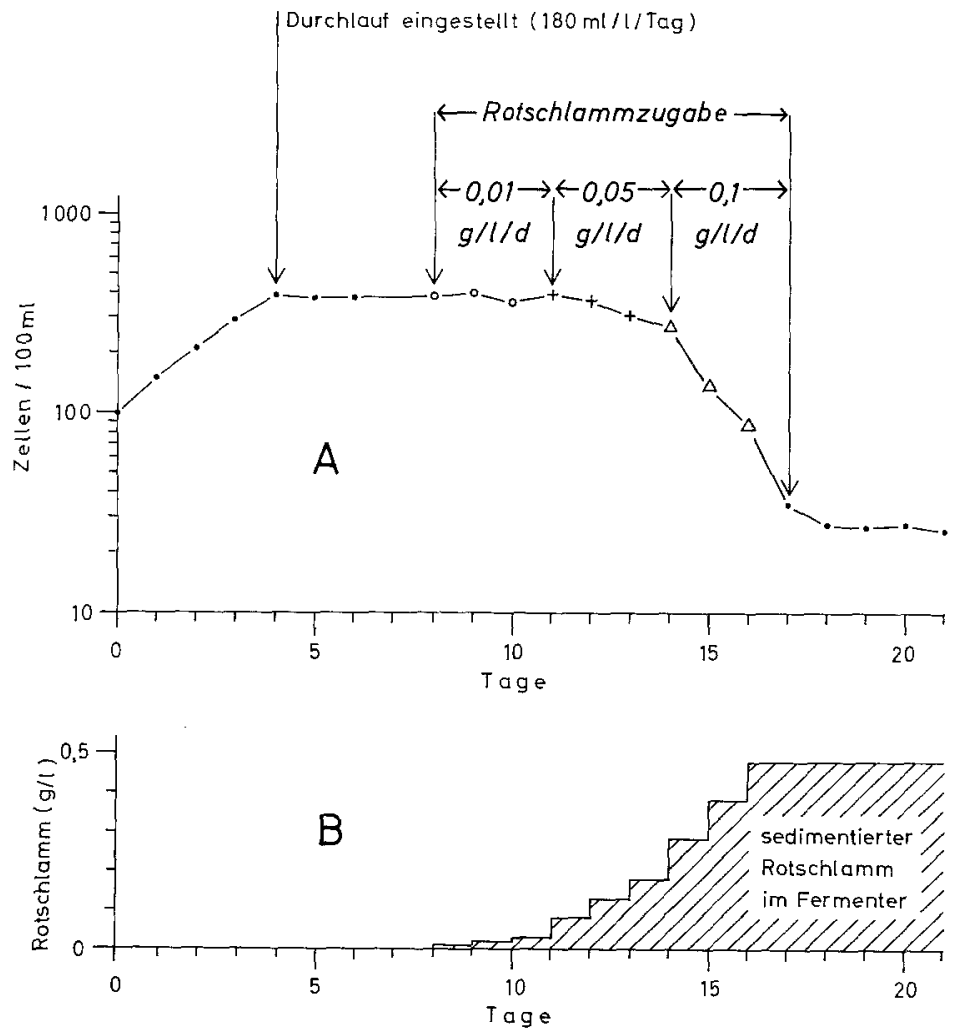

Abb. 12: Coscinodiscus granii. Kontinuierliche Kultur (Turbidostat) mit täglicher Zudosierung von frischem, fein suspendiertem Rotschlamm in Mengen von 0,01-0,1 g/l/Tag in einem 8-1Fermenter in Schreiberlösung unter Zusatz von Silikat. Der Rotschlamm wurde nach seiner Sedimentation nicht wieder aufgewirbelt. (A) Zelldichte der Kultur in Abhängigkeit vom Durchlauf des Kulturmediums und von den Rotschlammzugaben. (B) Zunahme des sedimentierten Rotschlamms am Boden des Versuchsgefäßes 
Zelldichte fiel daraufhin von ca. 400 auf ca. 290 Zellen/100 ml ab. Um diesen Effekt deutlicher zu machen, wurde die Dosis auf $0,1 \mathrm{~g} / 1 / \mathrm{Tag}$ gesteigert. Innerhalb der folgenden drei Tage fiel die Zelldichte dann auf ca. 35 Zellen $/ 100 \mathrm{ml}$ weiter ab. Nun wurden die Rotschlammzugaben eingestellt (17. Versuchstag). Die Dichte der Kultur sank am folgenden Tage noch bis auf den Wert von ca. 28 Zellen/100 ml ab. Dann fing sich die Kultur und hielt bei exponentiellem Wachstum wieder eine relativ konstante Zelldichte bei. Der Versuch wurde am 21. Tage beendet. In Abbildung 12B ist die Menge des während der Versuchszeit eingebrachten und überwiegend sedimentierten Rotschlammes dargestellt.

Der Versuch zeigt, daß sich eine tägliche „Beregnung“ mit frisch eingebrachtem, suspendiertem Rotschlamm in Mengen von $0,05 \mathrm{~g} / 1 / \mathrm{Tag}$ an negativ auszuwirken begann. Dieser Wert stimmt mit den Ergebnissen des Batch-Versuchs iberein, bei dem sich in der gleichen Konzentration eine starke Beeinträchtigung des Wachstums der Kulturen ergeben hatte. Ferner ist bemerkenswert, daß die Algen im Durchlaufsystem durchaus in der Lage waren, am Ende des Versuchs über einem Sediment von $0,48 \mathrm{~g} / 1$ Rotschlamm wieder in exponentielles Wachstum überzugehen, sofern die tägliche $\mathrm{Zu}$ fuhr frischen Rotschlammes eingestellt wurde.

\section{Chaetoceros socialis}

\section{Batch-Kultur}

Über Züchtungsexperimente mit dieser Alge wurde im Rahmen der Entwicklung eines Abwassertestverfahrens bereits an anderer Stelle berichtet (KAYSER, im Druck). Die als Beispiel für die Anwendung des Testverfahrens dargelegten ersten Ergebnisse eines Versuchs über den Einfluß von Rotschlamm sollen in dem hier gegebenen Zusammenhang nochmals ausführlicher besprochen werden.

$S t$ a ndversuch. Die Algen wurden in einer Serie von zehn 2-1-Gefäßen mit einer Anfangsdichte von 1000 Zellen/ml angesetzt. Rotschlamm wurde den Kulturen bei Versuchsbeginn in neun Konzentrationsstufen in Mengen von $0,001-10 \mathrm{~g} / 1$ einmalig zugesetzt und nach der Sedimentation nicht wieder aufgerihrt (vgl. p. 359). Die Kontrolle wuchs innerhalb von drei Tagen in exponentieller Phase bis zur maximalen Dichte von ca. $30000 \mathrm{Zellen} / \mathrm{ml}$ heran. In früheren und unter annähernd gleichen Bedingungen durchgeführten Versuchen konnte die maximale Zellzahl sogar Werte von ca. $45000 \mathrm{Zellen} / \mathrm{ml}$ erreichen. Diese und noch etwas höhere Werte (ca. $60000 \mathrm{Zellen}$ / $\mathrm{ml}$ ) wurden auch in den mit 0,01 und $0,001 \mathrm{~g} / 1$ Rotschlamm versetzten Kulturen dieser Serie erreicht. Eine Anfangsschädigung machte sich am ersten Tag nach der Rotschlammzugabe von $0,5 \mathrm{~g} / \mathrm{l}$ an bemerkbar. Sie führte bei $1 \mathrm{~g} / 1 \mathrm{zu}$ einem Absinken der Zellzahl auf ca. 600 Zellen/ml. Nach 5 und $10 \mathrm{~g} / 1$ Rotschlammzugabe sank die Zellzahl sogar auf 5 bzw. 3 Zellen/ml ab. Die überlebenden Zellen erholten sich jedoch anschließend und wuchsen in der über dem sedimentierten Rotschlamm stehenden Lösung in exponentieller Phase bis zu maximalen Zelldichten heran. Uber 5 g/l sedimentierten Rotschlammes erreichte die Dichte nach sieben. Tagen den Wert von ca. 55000 Zellen/ml. Rotschlamm wirkte sich demnach unter den gegebenen Versuchs- 
verhältnissen lediglich in einer konzentrationsabhängigen mechanischen Anfangsschädigung der Kulturen aus. Die letale Dosis wurde nicht erreicht.

\section{DISKUSSION}

Ein Uberblick über die Ergebnisse der Versuche zeigt, daß der Rotschlamm je nach Versuchsanordnung und verwendeter Algenart in sehr unterschiedlichen Konzentrationen wirksam wird. Die physikalisch-chemischen Prozesse, die unmittelbar nach der Zugabe des Rotschlammes zum Seewasser ablaufen, beeinflussen in entscheidender Weise den Beginn der Versuche. Flockungsvorgänge gelöster Aluminiumverbindungen aus dem Porenwasser des Schlammes, verbunden mit lockerer Koagulation feinster Rotschlammpartikel und anschließender Sedimentation führen zu einer bei allen Algenarten ausgeprägten Anfangsschädigung der Kulturen. Die über mehrere Tage im Seewasser gealterten Rotschlammpartikel zeigen eine vergleichsweise geringere Beeinflussung der Algen, die eine Wiedererholung der Kulturen erlauben kann. Dennoch wurde auch in dieser Phase eine deutliche Abhängigkeit des Algenwachstums von der Menge des in Schwebe befindlichen Rotschlammes festgestellt, wie ein Vergleich der Stand-, Schüttel- und Rührwerksversuche ergab. Die geringste Beeinflussung zeigten die Algen beim Wachstum über sedimentiertem Rotschlamm in überstehender Lösung. Die Konzentrationsgaben für Rotschlamm müssen dabei immer auf die anfänglich zugegebene Menge, nicht auf die in Schwebe befindlichen Partikel bezogen werden, da nach kurzer Zeit keinerlei homogene Verteilung im Versuchsgefäß vorliegt. Alle Konzentrationsangaben betreffen daher die zugegebene Anfangsdosis, unabhängig von ihrer späteren Sedimentation.

Die Versuche ergaben, daß die Schädlichkeit des Rotschlammes in erster Linie auf eine mechanische Wirkung der sedimentierenden Partikel zurückgeht. Dennoch ist eine toxische Wirkung des Schlammes, insbesondere der Porenlösung, nicht auszuschließen, wie der Versuch mit Gymnodinium splendens ergab, bei dem die Algen erst dem Versuch zugesetzt wurden, nachdem der Rotschlamm sedimentiert war (Abb. 10). Diese Wirkung setzte erst nach einer Woche deutlich ein. Auch für Prorocentrum micans konnte diese toxische Wirkung im Versuch in abdekantiertem Rotschlammüberstand hoher Konzentration nachgewiesen werden (Abb. 7). Es muß daher angenommen werden, daß eine toxische Langzeitwirkung von einer mechanischen Sofortwirkung überlagert wird.

Als empfindliche Arten erwiesen sich im Rahmen dieser Versuche Peridinium trochoideum, Gymnodinium splendens und Coscinodiscus granii. Etwas weniger empfindlich reagierte Prorocentrum micans, und die geringste Reaktion wurde bei Chaetoceros socialis registriert. Alle fünf verwendeten Testalgen müssen jedoch zu den relativ robusten und euryplastischen Formen im Plankton gerechnet werden. Nur solche Arten lassen sich im Labor unter einfachen Versuchsbedingungen und unter Verwendung von reinem oder nur mit wenigen Nährstoffen angereichertem Seewasser zu hinreichenden Zelldichten herankultivieren. Stenoplastische Vertreter des Planktons sind im Labor wesentlich schwieriger zu handhaben und können nur in kompliziert zusammengesetz- 
ten Nährlösungen gezüchtet werden. Im Hinblick auf die Reproduzierbarkeit und einfache Anwendungsmöglichkeit des Testverfahrens mußten daher raschwüchsige und in der Kultur leicht zu handhabende Formen ausgewählt werden. Bei der Beurteilung der Ergebnisse im Hinblick auf eine Verklappung größerer Mengen von Rotschlamm in freier See muß dieser Umstand jedoch berücksichtigt werden, da es im natürlichen Artengefügte in See Formen gibt, die wesentlich empfindlich reagieren.

In Tabelle 2 ist eine Übersicht über die ermittelten Grenzwerte für die verschiedenen Versuchsmethoden getrennt nach Algenarten gegeben. Coscinodiscus granii er weist sich mit einer Anfangsschädigung nach Zugabe von $0,005 \mathrm{~g} / 1$ Rotschlamm und einer Stagnation bei $0,05 \mathrm{~g} / \mathrm{l} \mathrm{im}$ Batch-Versuch als empfindlichste Form. Es folgt Peridinium trochoideum, die nach $0,01 \mathrm{~g} / 1$ täglicher Zugabe im kontinuierlichen Verfahren einen Rückgang der Zelldichte bis zum Absterben der Kultur zeigte. Die Anfangsschädigung im Batch-Verfahren lag bei $0,05 \mathrm{~g} / 1$ Rotschlammzugabe. Vergleichsweise Werte wurden auch für Gymnodinium splendens ermittelt, mit der jedoch nur zwei BatchVersuche durchgeführt wurden. Prorocentrum micans erweist sich mit einer Grenzkonzentration von $0,5 \mathrm{~g} / \mathrm{l} / \mathrm{Tag}$ im kontinuierlichen Verfahren als relativ robust, obwohl die Anfangsschädigungen im Batch-Verfahren schon bei $0,05 \mathrm{~g} / 1$ einsetzten. Als widerstandsfähigste Form erweist sich Chaetoceros socialis mit 'einer Anfangsschädigung bei $0,5 \mathrm{~g} / 1$ und einer guten Überlebensfähigkeit noch nach $10 \mathrm{~g} / 1$ Rotschlammzusatz im Batch-Verfahren.

Die unterschiedlichen Versuchsmethoden entsprechen durchaus den Bedingungen, die bei Verklappung größerer Mengen von Rotschlamm in See auftreten können. Die im kontinuierlichen Verfahren praktizierte tägliche Einbringung frischen Rotschlammes in das Kulturgefäß ist der punktförmigen Verklappung größerer Mengen von Rotschlamm in ein begrenztes Areal in See vergleichbar, in dem die Algen immer wieder mit frischem Material konfrontiert werden. Die in den Batch-Verfahren eingehaltene einmalige Zudosierung von Rotschlamm bei Versuchsbeginn entspricht der Einbringung von Rotschlamm in einen Wasserkörper, der nicht noch ein zweites Mal von frischem Rotschlamm getroffen wird, da er durch Tidenbewegung oder Verdriftung aus dem unmittelbaren Verklappungsareal herausbefördert wurde. Mittelbar bleibt die Rotschlammwirkung jedoch erhalten, da die alternden Partikel mitdriften bzw. sich über ein großes Areal als Sediment ausbreiten (vgl. Rosenthal et al. 1973). In der Praxis werden sich beide Fälle überlagern.

Ein weiterer, für die Ubertragung der Laborergebnisse auf die In-situ-Verhältnisse wichtiger Gesichtspunkt bedarf noch der Erwähnung. Im Labor wurde die Wirkung des Rotschlammes lediglich an exponentiell wachsenden Monokulturen untersucht. Diese Algen befanden sich unter optimalen Kulturbedingungen und standen unter keinerlei Konkurrenzdruck durch andere Algenarten oder durch planktonfressende Meerestiere. In freier See sind die Algen als Glieder natürlicher Lebensgemeinschaften durchaus nicht immer optimalen Bedingungen ausgesetzt und befinden sich daher nicht stets in der exponentiellen Wachstumsphase. Unter solchen Verhältnissen ist schon viel eher mit einer Schädigung der Art durch zusätzliche Belastungen, wie z. B. einer Rotschlammverklappung, zu rechnen. Diese Schädigung könnte eine Störung des biologischen Gleichgewichts nach sich ziehen, die im Rahmen des Schutzes der hohen See vor Abwasserschäden auf jeden Fall vermieden werden sollte. Die hier angegebe- 
nen Grenzwerte der ersten Schädigung einer Algenart können daher nur Richtwerte darstellen und sollten bei einer Übertragung auf die bei Rotschlammeinwirkung in See $\mathrm{zu}$ erwartende Gesamtsituation eher tiefer angesetzt werden.

Ein Vergleich der bisher in der Literatur erschienenen Ergebnisse über Rotschlammeinwirkung ergibt etwa folgendes Bild: Halsband \& Halsband (1971) untersuchten die Wirlsung von Rotschlamm an Batch-Kulturen von Dunaliella euchlora und Protococcus spec. Die Verfasser testeten lediglich Konzentrationen von 1, 2 und $5 \mathrm{~g} / \mathrm{l}$. Diese Algen wurden in zwei Serien angesetzt und einmal dem Rotschlamm direkt zugesetzt; zum anderen Male wurde der Rotschlamm vor Versuchsbeginn abfiltriert und die Algen erst dann dem Filtrat eingeimptt. Die Versuche in der ersten Serie ergaben eine höhere Empfindlichkeit der Algen als in der zweiten Serie. Als „Erträglichkeitsgrenze“ wird die Konzentration von $1 \mathrm{~g} / 1$ angegeben. Dieser relativ hohe Wert erklärt sich zum einen aus den verwendeten Algenarten, zum anderen aus der Methode, die insofern von den hier beschriebenen Versuchen abweicht, als die Algen erst nach Eingabe des Rotschlammes den Versuchen zugesetzt wurden. Eine entsprechende Versuchsanordnung wurde hier nur im Überstandsversuch mit Gymnodinium splendens bzw. in dem mit dekantiertem Rotschlammüberstand gefahrenen Versuch mit Prorocentrum micans eingehalten. Diese Versuche ergaben ebenfalls relativ geringe Beeinträchtigungen der Algen.

Dethlefsen (1972a) ergänzte die Versuche von Halsband \& Halsband. Er untersuchte die Wirkung von Rotschlamm in Konzentrationen von $0,5-50 \mathrm{~g} / 1$ an gesättigten Grünalgenpopulationen hoher Dichte (stationäre Phase), indem er die Algen (Monadinen) mit den entsprechenden Rotschlammkonzentrationen zusammen ansetzte und den Abfall der Algendichte über einen Zeitraum von 26 bzw. 28 Tagen nach Filtrat photometrisch bestimmte. In allen von ihm untersuchten Rotschlammkonzentrationen stellte er eine Beeinträchtigung der Populationsdichte im Vergleich zur Kontrolle fest, ohne die subletalen und letalen Grenzwerte zu erreichen.

Die Untersuchungen über Schadwirkungen an Meerestieren nehmen in der Literatur bereits einen relativ breiten Raum ein. Es können daher nur die wichtigsten Ergebnisse kurz referiert werden. Greve \& KINNE (1971) konnten nachweisen, daß die Nahrungsaufnahme der Rippenqualle Pleurobrachia pileus bei $0,1 \mathrm{ml} / 1$ Rotschlamm leicht gestört wurde. Krebse der Art Calanus belgolandicus zeigten nach PfaffenHöfer $(1971,1972)$ schon in Konzentrationen von $0,01 \mathrm{ml} / 1$ Rotschlamm Anstieg der Sterblichkeit und Abnahme der Wachstumsrate. HALSBAND (1971) gibt als Erträglichkeitsgrenze für Garnelen (Crangon crangon) die Konzentration von 0,2 g/1 Rotschlamm an. Kiemenverklebung durch Rotschlammpartikel wird als Hauptursache für die Beeinträchtigung von Garnelen und Fischen angegeben (HaLsband \& Halsband 1971). Sie untersuchten physiologische Reaktionen bei der Scholle (Pleuronectes platessa) und beim Aal (Anguilla anguilla). Rosenthal (1971) führte Experimente an Embryonen und Larven des Herings (Clupea barengus) durch. Er beobachtete Verklebungen der Eioberflächen durch Rotschlammpartikel, die den Gasaustausch beeinträchigten und bei fortschreitender Entwicklung der Fischembryonen auf Grund unzureichender Sauerstoffversorgung eine hohe Sterblichkeit der Keime verursachten. Ferner zeigte es sich, daß die vollständige Verklebung der Eioberfläche weitgehend unabhängig von der Ausgangskonzentration der Rotschlammpartikel ist. Maul und Schlund von Herings- 
larven wurden durch Rotschlamm verklebt. Wie aus anderen, unveröffentlichten Arbeiten hervorgeht (Gutachten zur Rotschlammverklappung 1972), werden Rotschlammpartikel von vielen filtrierenden Meeresorganismen zwangsläufig mit der Nahrung zusammen aufgenommen und beeinträchtigen dadurch deren Stoff wechsel.

Von französischer Seite wurde ein wissenschaftliches Gutachten über die Einleitung von Rotschlamm unweit der Bucht von Cassis (nahe bei Marseille) erstellt (GAUGENHEIM et al., 1966). In diesem Gebiet wird der Rotschlamm seit 1966 mit Hilfe einer $7 \mathrm{~km}$ langen Pipeline in einer Tiefe von $350 \mathrm{~m}$ in einen untermeerischen Canyon eingeleitet, der sich mit einer schmalen Rinne bis auf Tiefen von 1800 bis $2000 \mathrm{~m}$ seewärts öffnet. Der Rotschlamm kann hier ungehindert in die Tiefsee abfließen, ohne sich in flacheren Gewässern auszubreiten. Bourcier (1969) konnte nachweisen, daß im Einleitungszentrum dieses Areals eine tote Zone entstanden ist und an den Rändern des Ausbreitungsfeldes im wesentlichen eine Verarmung des natürlichen Artenspektrums zugunsten einiger widerstandsfähiger Organismen eingetreten ist.

In England wurde Rotschlamm über einen Zeitraum von 6 Jahren mit einer Menge von insgesamt $263000 \mathrm{t}$ in den Bristolkanal eingeleitet, ohne daß akute Schäden im Einleitungsgebiet festgestellt worden wären (DAvis 1972). In dem $8,5 \mathrm{~km}$ vor der Küste gelegenen Einleitungsgebiet herrschte jedoch eine starke Gezeitenströmung bei einem Tidenhub von maximal $12 \mathrm{~m}$. Der vor der Verklappung in Sammelbehältern gelagerte Rotschlamm wurde jeweils nur bei Hochwasser eingebracht und durch die Strömung rasch seewärts transportiert.

Die an den deutschen Rotschlamm-Untersuchungen beteiligten Institutionen (Biologische Anstalt Felgoland, Bundesforschungsanstalt für Fischerei, Deutsches Hydrographisches Institut und Institut für Meeresforschung, Bremerhaven) stellten an Hand der bisher gewonnenen Ergebnisse in einer gemeinsamen gutachtlichen Stellungnahme fest, daß bei ständigem Einbringen von Rotschlamm in die südliche Nordsee, die ein Flachmeer mit geringer Wassertiefe darstellt, mit einer Beeinträchtigung der Lebensgemeinschaften und einer nachhaltigen Störung des biologischen Gleichgewichts gerechnet werden muß. Auch die hier beschriebenen Ergebnisse über die Wirkung von Rotschlamm auf marine Planktonalgen bekräftigen diese Feststellung. Diese ursprünglich im Rahmen des gemeinsamen Gutachtens begonnenen Arbeiten wurden nach dessen Fertigstellung noch beträchtlich erweitert, ehe sie hier dargelegt wurden. Auf Grund der bei der Probeverklappung in freier See nachgewiesenen unerwartet raschen Ausbreitung des Rotschlammes weit über das Verklappungsgebiet hinaus wird aus fischereilicher Sicht eine Beeinträchtigung des Lebensraumes der Nutzfischbestände gesehen, so daß bei kontinuierlicher Einbringung mit einer Schädigung der Fischerei gerednet werden müßte. Die Einbringung von Rotschlamm in die südliche Nordsee wurde daher abgelehnt.

\section{ZUSAMMENFASSUNG}

1. Rotschlamm ist ein bei der Aluminiumgewinnung aus Bauxit anfallendes Abfallprodukt. Der Antrag eines Industrieunternehmens auf Verklappung von ca. 800000 t Rotschlamm (Naßgewicht) pro Jahr in der Nordsee gab den unmittel- 
baren Anlaß zur Aufnahme von Laborversuchen über die Wirkung auf marine Planktonalgen. Als Testformen dienten die Dinoflagellaten Peridinium trochoideum, Gymnodinium splendens und Prorocentrum micans sowie die Diatomeen Coscinodiscus granii und Chatoceros socialis. Die Algen wurden sowohl im Batch-Verfahren als auch im kontinuierlichen Verfabren nach dem Turbidostatprinzip gezüchtet.

2. Rotschlamm wurde den Kulturen in Batch-Verfahren einmalig bei Versuchsbeginn fein suspendiert zugesetzt und je nach Versuchsanordnung entweder der Sedimentation überlassen oder durch regelmäßiges Aufschütteln bzw. vermittels eines Rührwerkes soweit wie möglich in Turbulenz gehalten. Im kontinuierlichen Verfahren wurde den Kulturen während der gesamten Versuchsdauer täglich frischer Rotschlamm zudosiert und der Sedimentation überlassen. Die Menge des zugegebenen Rotschlammes variierte je nach Versuchsanordnung zwischen 0,001 und $50 \mathrm{~g}(\mathrm{Naß}$ gewicht)/l Seewasser.

3. Als Kriterien einer Schädigung dienten die Beeinflussung der Vermehrungsrate der Algen und die maximal erreichbare Zelldichte der Kulturen. Zugleich wurden die letalen Grenzkonzentrationen bestimmt.

4. Die Testversuche zeigten, daß die Empfindlichkeit gegenüber Rotschlamm wesentlich von der verwendeten Algenart und von der jeweiligen Versuchsanordnung abhängt. Reversible Anfangsschädigungen wurden im Batch-Verfahren bei $0,005 \mathrm{~g} / 1$ (Coscinodiscus granii), 0,01 $\mathrm{g} / 1$ (Gymnodinium splendens), $0,05 \mathrm{~g} / 1$ (Peridinium trochoideum und Prorocentrum micans) und $0,5 \mathrm{~g} / 1$ (Chaetoceros socialis) nachgewiesen. Irreversible Schädigung führte im kontinuierlichen Verfahren bei täglicher Zudosierung des Rotschlammes in Mengen von $0,01 \mathrm{~g} / 1$ (Peridinium trochoideum), 0,05 g/1 (Coscinodiscus granii) und $0,5 \mathrm{~g} / 1$ (Prorocentrum micans) zum Absterben der Kulturen.

5. Die Anfangsschädigung ist in erster Linie auf mechanische Wirkung unmittelbar nach Zugabe des frischen Rotschlammes zum Meerwasser zurückzuführen. Die Partikel sedimentieren unter lockerer Koagulation und reißen die Algen mit sich. Gealterte Rotschlammpartikel erweisen sich als weniger schädlich. In höheren Konzentrationen kann eine toxische Wirkung hinzukommen $(0,5 \mathrm{~g} / \mathrm{L}$ Rotschlamm bei Gymnodinium splendens), die sich jedoch erst nach längerer Versuchsdauer bemerkbar macht.

6. Da die verwendeten Testalgen den relativ robusten Planktonformen zugehören, ist bei einer Übertragung der Ergebnisse auf die in See eintretende Gesamtsituation damit zu rechnen, daß viele andere Planktonalgen gegenüber einer Rotschlammverklappung noch empfindlicher reagieren.

7. In einer Literaturübersicht werden die von anderen Autoren an repräsentativen Gliedern der Nahrungskette (Algen, Fischnährtiere und Fische) ermittelten wichtigsten Ergebnisse von Rotschlammversuchen kurz referiert und den vorliegenden Ergebnissen gegenübergestellt. In einer gemeinsamen Stellungnahme mehrerer bundesdeutscher Institute wird aus den angeführten Untersuchungen die Schlußfolgerung gezogen, daß einer Einbringung von Rotschlamm in die Nordsee nicht zugestimmt werden kann. 
Danksagungen. Der Deutschen Forschungsgemeinschaft wird für die finanzielle Unterstützung des Forschungsvorhabens gedankt. Meinen technischen Assistentinnen Frl. I. SCHUmacher und Frau U. Michels danke ich für die Hilfe bei der Durchführung dieser Arbeit.

\section{ZITIERTE LITERATUR}

Apelt, G., 1971. Untersuchungen über die Boden-Meiofauna im Rotschlamm-Verklappungsgebiet nordwestlich der Weißen Bank. 3. Kolloquium zum Schwerpunktprogramm der DFG "Litoralforschung, Abwasser in Küstennähe“, Bremerhaven.

Bourcier, M., 1969. Ecoulement des Boues-rouges dans le canyon de la cassidaigne. Thetys 1 , $779-782$.

Davis, V. E. Erfahrungen bei der Versenkung von Rotschlamm im Meer. Vortrag auf der Hauptversammlung d. Ges. Deutscher Metallhütten- und Bergleute e. V., Stuttgart, 27. 4. 1972. (Im Druck).

Dethlefsen, V., 1972a. Über den Einfluß von Rotschlamm auf die Besiedlungsdichte von Grünalgen. Arch. FischWiss. 23, 68-72.

- 1972b. On the effects of red mud on marine organisms. Counc. Meet. int. Counc. Explor. Sea (= C.M. - I.C.E.S.) E 7, 1-7.

- \& Rosenthal, H., 1973. Problems with dumping of red mud in shallow waters. A critical review of selected literature. Aquaculture 2, 267-280.

Gaugenheim, A., Chedin, J., Drach, P., Lacombe, H., Larras, J. \& Vincotte, J., 1966. Rapport de la Commission d'éxperts chargés d'examiner les problèmes posés par le projekt de deversement du boues rouges dans la baie de Cassis, 1er Mars 1965. J. off. Ministêre des Traveaux publics et des Transports, Ministêre de l'Industrie 1966, 1-19.

GREVE, W. \& KinNE, O., 1971. Über die Reaktion einiger Vertreter des vagilen Makrobenthos auf die Konfrontation mit Rotschlamm. 3. Kolloquium zum Schwerpunktprogramm der DFG „Litoralforschung, Abwasser in Küstennähe“, Bremerhaven.

Halsband, E., 1971. Die physiologische Wirkung von Rotschlamm, Kupfersulfat, Quecksilberbromid und Zinkchlorid auf Seewasserfische. 3. Kolloquium zum Schwerpunktprogramm der DFG „Litoralforschung, Abwasser in Küstennähe“, Bremerhaven.

- \& Halsband, I., 1971. Physiologische Studien zur Ermittlung des Schädlichkeitsgrades von „Rotschlamm“ in Seewasser. Wass. Luft Betr. 15, 268-273.

KAYSER, H., 1969. Züchtungsexperimente an zwei marinen Flagellaten (Dinophyta) und ihre Anwendung im toxikologischen Abwassertest. Helgoländer wiss. Meeresunters. 19, 21-44.

- 1970a. Experimental-ecological investigation on Pbaeocystis poucheti (Faptophyceae): Cultivation and waste water test. Helgoländer wiss. Meeresunters. 20, 195-212.

- 1970b. Pollution of the North Sea and rearing experiments on marine phytoflagellates as an indication of resultant toxicity. In: Advances in water pollution research. Ed. by S. H. Jenkins. Pergamon Press, Oxford, 2, Paper III-2.

- 1971a. Gewässer und Pestizide, Fachgespräch im Inst. f. Wasser-, Boden- und Lufthygiene, Berlin. SchrReihe Ver. Wass.- Boden- u. Lufthyg. 34, 147-148.

- 1971b. Abwasserteste an Kulturen mariner Planktonalgen. 3. Kolloquium zum Schwerpunktprogramm der DFG „Litoralforschung, Abwasser in Küstennähe“, Bremerhaven.

- Produktivitätsmessungen an Phytoplanktonorganismen aus Küstengewässern als Standardmethode für einen Abwassertest. Thalassia jugosl. (Im Druck.)

NAukE, H. Rotschlammverklappungen in der Nordsee - geologische Ergebnisse eines Großversuchs. Dt. hydrogr. Z. (Im Druck.)

PAFFenнöFER, G.-A., 1971. Einfluß von „Rotschlamm" auf Mortalität und Körpergewicht von Jugendstadien des marinen planktischen Copepoden Calanus helgolandicus. Naturwissenschaften $\mathbf{5 8}, 625$.

- 1972. The effects of suspended "red mud" on mortality, body weight, and growth of the marine planktonic copepod, Calanus belgolandicus. Water Air Soil Poll. 1, 314-321.

Rachor, E., 1971. Der Bestand der Makro-Bodenfauna in einem durch Rotschlammverklap- 
pung beeinflußten Gebiet in der Deutschen Bucht. 3. Kolloquium zum Schwerpunktprogramm der DFG „Litoralforschung, Abwasser in Küstennähe“, Bremerhaven.

Rosenthal, H., 1971. Wirkungen von "Rotschlamm“ auf Embryonen und Larven des Herings, Clupea harengus. Helgoländer wiss. Meeresunters, 22, 366-376.

- Detrilefsen, V. \& Trews, K., 1973. Rotschlamm in der Nordsee? Umschau 73, 118-121.

Weichart, G., 1969. Industrielle Abfallstoffe gefährden die Nordsee. Umschau 69, 605-611.

Anschrift des Autors: Dr. H. Kayser

Biologische Anstalt Helgoland

(Meeresstation)

2192 Helgoland

Bundesrepublik Deutschland 